\title{
Hydrodynamic model approach to the formation of plasmonic wakes in graphene
}

\author{
Chaves, A. J.; Peres, N. M. R.; Smirnov, G.; Mortensen, N. Asger
}

Published in:

Physical Review B

Link to article, DOI:

10.1103/PhysRevB.96.195438

Publication date:

2017

Document Version

Publisher's PDF, also known as Version of record

Link back to DTU Orbit

Citation (APA):

Chaves, A. J., Peres, N. M. R., Smirnov, G., \& Mortensen, N. A. (2017). Hydrodynamic model approach to the formation of plasmonic wakes in graphene. Physical Review B, 96(19), [195438].

https://doi.org/10.1103/PhysRevB.96.195438

\section{General rights}

Copyright and moral rights for the publications made accessible in the public portal are retained by the authors and/or other copyright owners and it is a condition of accessing publications that users recognise and abide by the legal requirements associated with these rights.

- Users may download and print one copy of any publication from the public portal for the purpose of private study or research.

- You may not further distribute the material or use it for any profit-making activity or commercial gain

- You may freely distribute the URL identifying the publication in the public portal 


\title{
Hydrodynamic model approach to the formation of plasmonic wakes in graphene
}

\author{
A. J. Chaves, ${ }^{1}$ N. M. R. Peres, ${ }^{1}$ G. Smirnov, ${ }^{2}$ and N. Asger Mortensen ${ }^{3,4,5}$ \\ ${ }^{1}$ Department and Center of Physics, and QuantaLab, University of Minho, Campus of Gualtar, PT-4710-374, Braga, Portugal \\ ${ }^{2}$ Department of Mathematics and Applications, and Center of Physics, University of Minho, \\ Campus of Gualtar, PT-4710-374, Braga, Portugal \\ ${ }^{3}$ Center for Nano Optics, University of Southern Denmark, Campusvej 55, DK-5230 Odense M, Denmark \\ ${ }^{4}$ Center for Nanostructured Graphene, Technical University of Denmark, Orsteds Plads 343, DK-2800 Kongens Lyngby, Denmark \\ ${ }^{5}$ Danish Institute for Advanced Study, University of Southern Denmark, Campusvej 55, DK-5230 Odense M, Denmark
}

(Received 18 August 2017; revised manuscript received 14 November 2017; published 28 November 2017)

\begin{abstract}
Using the hydrodynamic model in the electrostatic approximation, we describe the formation of graphene surface plasmons when a nearby charge is in motion either perpendicular or parallel to a graphene sheet. In the first case, the electron-energy loss (EEL) spectrum of the electron is computed, showing that the resonances in the spectrum are linked to the frequency of the graphene surface plasmons. In the second case, we discuss the formation of plasmonic wakes due to the dragging of the surface plasmons induced by the motion of the charge. This effect is similar to Coulomb drag between two electron gases at a distance from each other. We derive simple expressions for the electrostatic potential induced by the moving charge on graphene. We show that there is a transition from a Mach-type wake at high speeds to a Kelvin-type wake at low ones and identify the Froude number for plasmonic wakes. We show that the Froude number can be controlled externally by tunning both the Fermi energy in graphene and the dielectric function of the environment, a situation with no parallel in ship wakes. Using EEL, we propose a source of graphene plasmons, based on a graphene drum built in a metallic waveguide and activated by an electron beam created by the tip of an electronic microscope. We also introduce the notion of a plasmonic billiard.
\end{abstract}

DOI: 10.1103/PhysRevB.96.195438

\section{INTRODUCTION}

The hydrodynamic model $[1,2]$ for plasmonics is a macroscopic approach to a microscopic problem, as was well noted by Ciracì et al. [3] (see also Ref. [4]). This model combines Maxwell's equations, Euler's equation of hydrodynamics supplemented with a term due to the statistical pressure of an electron gas, and the continuity equation. This set of equations is used for describing the nonlocal optical response of either a metallic interface [5-7] or a metallic nanostructure [8,9]. The model can be applied both to 3D [10], 2D [11-13], or 1D metallic structures $[9,14,15]$. In the latter case, the situation of two metallic nanoparticles in close proximity is rather important as it allows us to probe electromagnetic interactions between them down to the separation of few atoms $[16,17]$. Indeed, whereas an electromagnetic local description of the dimer optical properties predicts a divergent enhancement of the electromagnetic energy density in the gap region between the two nanoparticles, a nonlocal description predicts a reduction of the field enhancement when the two particle are at atomic distances from each other, in agreement with the experimental observations [16,17].

In the past six years, graphene has emerged as a new platform for studying plasmonic effects in the THz and mid-IR, a spectral range where noble metal plasmons show poor spatial confinement. Since the hydrodynamic model can be applied to the 2D electron gas [12], a natural question arises whether graphene, which supports a massless electron gas, can also be described by the hydrodynamic model. Müller et al. have shown that the massless electron gas in graphene behaves as a nearly perfect fluid [18] with the electronic motion described by the Navier-Stokes equation, from which Euler's equation follows. This model for electronic motion has subsequently been applied to the characterization of the conductivity of graphene [19] as well as to the characterization of its plasmonic properties [11].

One of the merits of the hydrodynamic model [20,21] is permitting the inclusion of nonlocal effects in the plasmonic response of very small metallic nanostructures without much computational burden. Within this model, nonlocality appears due to the dependence of the statistical pressure on the position-dependent electronic density, and Coulomb interactions are included via the coupling of Euler's equation with Maxwell's equations (or via Poisson's equation in an electrostatic calculation). Nonlocal effects emerge when the size of the nanostructures becomes small enough for coarse graining of the electronic charge no longer holds [16]. These effects have also impact in the optical properties of metallic gratings [22]. This happen when the wavelength of the surface plasmon in the metal is smaller than typical size of the nanostructures. Typically, the condition $q c / \omega_{p} \gg 1$, where $q, c$, and $\omega_{p}$ are the wave number of the surface plasmon, the speed of light in vacuum, and the plasma frequency of the metal, has to be fulfilled for nonlocality to play an important role in the optical spectrum of the system. Physically, nonlocality arises due to the smearing of the electronic charge when probed down to the nanoscale [23]. As a consequence, the screening of the electromagnetic fields becomes less efficient when compared to the local calculation prediction. Given this, a simple model [7] for a nonlocal metal was recently introduced which allows much analytical progress.

For graphene, the criterion for nonlocality to be important is $q c /\left(k_{F} v_{F}\right) \gg 1$, with $k_{F}$ and $v_{F}$ being the Fermi wave number and Fermi speed, respectively (clearly, the system will be highly nonlocal near the graphene neutrality point). For graphene near a metal, the dispersion is strongly dependent on distance between the two systems. Taking a metal-graphene 
distance of about $1.5 \mathrm{~nm}$, the graphene-metal plasmons can appear in the mid-IR with a wave number of the order of $200 \mu \mathrm{m}^{-1}$ (corresponding to a $\lambda_{\text {spp }}=2 \pi / q \approx 30 \mathrm{~nm}$ ). Considering a Fermi energy of graphene of about $E_{F}=$ $0.4 \mathrm{eV}$, the previous condition gives $q c /\left(k_{F} v_{F}\right) \sim 100$, thus placing graphene in the strong nonlocal regime. With these conditions, this means that the optical properties of graphene, such as its optical conductivity, have to be taken as a function of both frequency $\omega$ and wave vector $\mathbf{k}$ in order to account for both temporal and spatial dispersion. Recently, it became possible to retrieve experimentally the nonlocal optical conductivity of graphene using acoustic graphene plasmons [24], introducing a test for the accuracy of many-body calculations.

In this paper, nonlocality in graphene is taken into account at the level of the hydrodynamic model, considering a quantum pressure term that is spatially dependent. Basically, hydrodynamics is the small-wave number correction to the local description, and by carefully expanding the response function emerging from the random-phase approximation (RPA), we in general find a two-fluid model to account for both the interband and intraband effects, which both exhibit spatial dispersion, but with different nonlocal parameters [25]. In this paper we neglect the interband contribution, since in the frequency window we are interested in, these type of transitions are blocked by Pauli's principle.

The remaining of the paper is organized as follows. In Sec. II, we study the hydrodynamic model with external potentials due to external charge densities. This sets the basic equations for describing the effect of moving charges on graphene electronic properties. In Sec. III, the effect of moving charges (see Fig. 1) in the induction of plasmonic wakes at the surface of graphene is studied. In order to discuss plasmonic effects in graphene nanostructures, we consider in Sec. IV the excitation of localized graphene plasmons by an electron in motion, impinging perpendicularly to a nanorectangle of graphene located in a metallic waveguide. Finally, in Sec. V, we present a short overview of the paper, discuss possible extensions of this work, and introduce the concepts of Froude number for plasmonic wakes and of plasmonic billiards. In Appendix A, we present a short derivation to Euler's equation of hydrodynamics starting from Boltzmann equation. In Appendix B, the hydrodynamic model for graphene is presented and the derivation of the spectrum of the graphene plasmons is given.

\section{2D HYDRODYNAMIC MODEL IN THE PRESENCE OF EXTERNAL POTENTIALS}

The hydrodynamic model of the electromagnetic response of an electron gas couples Euler's equation (see Appendix A for a derivation starting with Boltzmann's equation) to Maxwell's equations $[20,21,26,27]$. Within this model the spectrum of the surface plasmon-polaritons easily follows, as shown in Appendix B (the reader should consult this appendix for details on the notation used below). To emphasize the $2 \mathrm{D}$ nature of the problem, we will in the following introduce $\mathbf{r}=\left(\mathbf{r}_{\|}, z\right)$ with $\mathbf{r}_{\|}$ being a $2 \mathrm{D}$ position vector in the plane of the graphene $(z=0)$, while $z$ is in the direction perpendicular to the graphene layer.

In this section, we follow Fetter [12] for the calculation of the response of the electron gas to external potentials. Let us

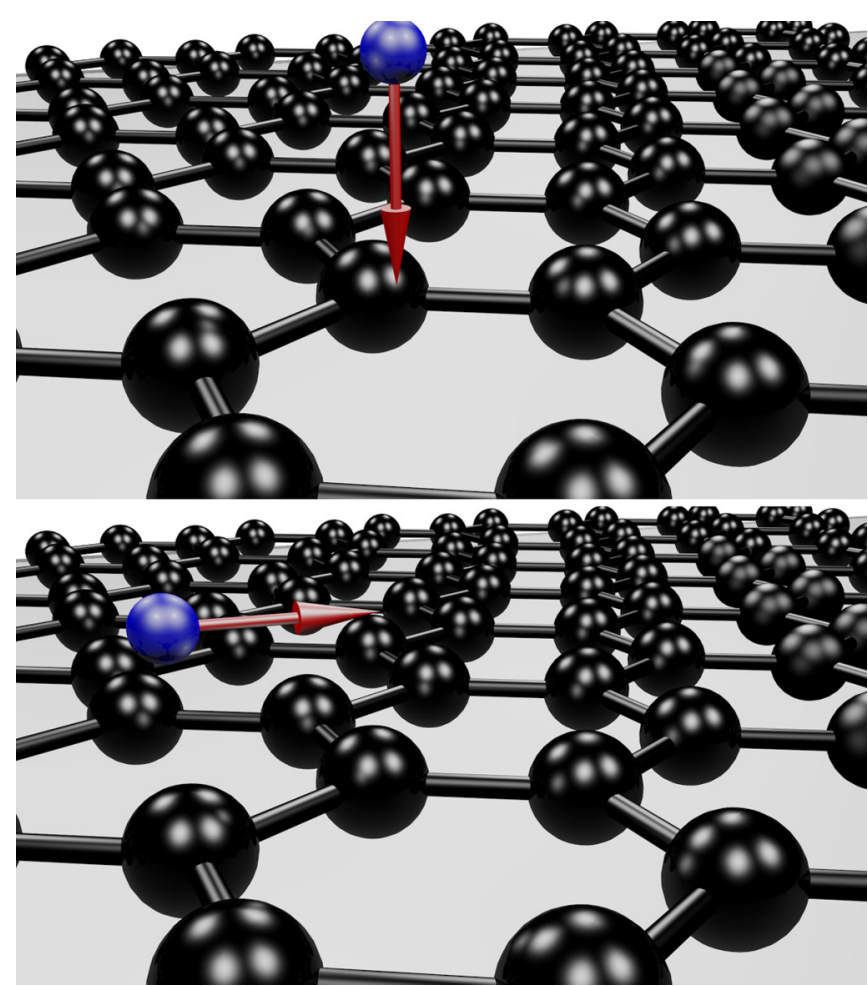

FIG. 1. System considered in this paper: a charge is moving relatively to graphene either perpendicular (top) or parallel (bottom) to it. The effect of the interaction of the charge with the electron gas in graphene is studied using the hydrodynamic model, which has built in nonlocal corrections due to the statistical pressure of the electron gas. The charge induces surface plasmons in graphene which can be probed by EEL spectroscopy.

consider the additional presence of external electrostatic forces acting on the electron gas. This is accounted for by adding extra terms to both the hydrodynamic equation and Poisson's equation (see Appendixes A and B). These two equations are modified as

$$
\begin{gathered}
\frac{\partial \mathbf{v}}{\partial t}=\frac{e v_{F}}{\hbar k_{F}} \nabla\left[\phi_{1}\left(\mathbf{r}_{\|}, 0\right)+\phi_{\mathrm{ex}}\left(\mathbf{r}_{\|}, 0\right)\right]-\frac{v_{F}^{2}}{2 n_{0}} \nabla n_{1}\left(\mathbf{r}_{\|}\right) \\
\nabla^{2}\left[\phi_{1}(\mathbf{r})+\phi_{\mathrm{ex}}(\mathbf{r})\right]=-\frac{e}{\epsilon_{0}} \rho_{\mathrm{ex}}(\mathbf{r})+\frac{e}{\epsilon_{0}} \delta(z) n_{1}\left(\mathbf{r}_{\|}, 0\right),
\end{gathered}
$$

where $\phi_{\mathrm{ex}}\left(\mathbf{r}_{\|}, z\right)$ is the external potential due to the external forces, $\rho_{\mathrm{ex}}(\mathbf{k}, z, \omega)$ is the volume density of external charges, and the continuity equation is unchanged by the presence of the additional potentials. Next, we introduce the Fourier transform of the different quantities, leading to

$$
-i \omega \mathbf{v}(\mathbf{k}, \omega)=\frac{e v_{F}}{\hbar k_{F}} i \mathbf{k} \phi(\mathbf{k}, 0, \omega)-\frac{v_{F}^{2}}{2 n_{0}} i \mathbf{k} n_{1}(\mathbf{k}, \omega)
$$

for Euler's equation and

$$
\begin{aligned}
\left(\frac{\partial^{2}}{\partial z^{2}}-k^{2}\right) \phi(\mathbf{k}, z, \omega)= & -\frac{e}{\epsilon_{0}} \rho_{\mathrm{ex}}(\mathbf{k}, z, \omega) \\
& +\frac{e}{\epsilon_{0}} \delta(z) n_{1}(\mathbf{k}, \omega)
\end{aligned}
$$


for Poisson's equation, where $\phi(\mathbf{k}, z, \omega)=\phi_{1}(\mathbf{k}, z, \omega)+$ $\phi_{\mathrm{ex}}(\mathbf{k}, z, \omega)$ [the continuity equation is unchanged: $0=$ $\left.-i \omega n_{1}(\mathbf{k}, \omega)+n_{0} i \mathbf{k} \cdot \mathbf{v}(\mathbf{k}, \omega)\right]$. For solving Eq. (2b), we use the Green's function method. The free space Green's function is defined as

$$
\left(\frac{\partial^{2}}{\partial z^{2}}-k^{2}\right) g\left(\mathbf{k}, z-z^{\prime}, \omega\right)=-\delta\left(z-z^{\prime}\right),
$$

from where it follows that

$$
\phi(\mathbf{k}, z, \omega)=\int d z^{\prime} g\left(\mathbf{k}, z-z^{\prime}, \omega\right) \rho\left(\mathbf{k}, z^{\prime}, \omega\right)
$$

where

$$
\rho(\mathbf{k}, z, \omega)=-\frac{e}{\epsilon_{0}}\left[\rho_{\mathrm{ex}}(\mathbf{k}, z, \omega)-\frac{e}{\epsilon_{0}} \delta(z) n_{1}(\mathbf{k}, \omega)\right] .
$$

The solution of Eq. (3) is well known and reads $g(\mathbf{k}, z-$ $\left.z^{\prime}, \omega\right)=\exp \left(-k\left|z-z^{\prime}\right|\right) /(2 k)$. As a consequence, the potential reads

$$
\begin{aligned}
\phi(\mathbf{k}, z, \omega)= & \int d z^{\prime} \frac{e}{2 k \epsilon_{0}} e^{-k\left|z-z^{\prime}\right|} \rho_{\mathrm{ex}}\left(\mathbf{k}, z^{\prime}, \omega\right) \\
& -\frac{e}{2 k \epsilon_{0}} e^{-k|z|} n_{1}(\mathbf{k}, \omega),
\end{aligned}
$$

which we write compactly as $\phi(\mathbf{k}, z, \omega)=\Phi_{\mathrm{ex}}(\mathbf{k}, z, \omega)-$ $\Phi_{2 D, 1}(\mathbf{k}, z, \omega)$, where

$$
\Phi_{\mathrm{ex}}(\mathbf{k}, z, \omega)=\int d z^{\prime} \frac{e}{2 k \epsilon_{0}} e^{-k\left|z-z^{\prime}\right|} \rho_{\mathrm{ex}}\left(\mathbf{k}, z^{\prime}, \omega\right) .
$$

Using this result in the hydrodynamic equation, we obtain

$$
\begin{aligned}
\omega \mathbf{v}(\mathbf{k}, \omega)= & \frac{e}{m} \mathbf{k}\left[\Phi_{2 D, 1}(\mathbf{k}, 0, \omega)-\Phi_{\mathrm{ex}}(\mathbf{k}, 0, \omega)\right] \\
& +\frac{v_{F}^{2}}{2 n_{0}} \mathbf{k} n_{1}(\mathbf{k}, \omega) .
\end{aligned}
$$

From the previous equation, we obtain the velocity, which we plug in the continuity equation, that can be solved for $n_{1}(\mathbf{k}, \omega)$, and the induced potential is given by

$$
\begin{aligned}
\Phi_{\text {in }}(\mathbf{k}, z, \omega) & =-\Phi_{2 D, 1}(\mathbf{k}, z, \omega) \\
& =\frac{n_{0} e^{3}}{4 \epsilon_{0}^{2} m_{g}} \frac{e^{-k|z|}}{\omega^{2}-\omega_{\mathrm{spp}}^{2}} \int d z^{\prime} e^{-k\left|z^{\prime}\right|} \rho_{\mathrm{ex}}\left(\mathbf{k}, z^{\prime}, \omega\right) .
\end{aligned}
$$

For computing these quantities in real space an inverse Fourier transform has to be performed.

\section{INDUCED ELECTROSTATIC POTENTIAL DUE TO A MOVING CHARGE}

Next we consider two applications of the central results obtained in the previous section. We shall consider the calculation of the induced electrostatic potential $\Phi_{\text {in }}\left(\mathbf{r}_{\|}, z, \omega\right)$ and induced electric field in graphene, $\mathbf{E}\left(\mathbf{r}_{\|}, z, \omega\right)=-\nabla \Phi_{\text {in }}\left(\mathbf{r}_{\|}, z, \omega\right)$, due to a charge $Z e$ moving at the speed $v$. We consider two cases:

$$
\begin{aligned}
& \rho_{\mathrm{ex}}\left(\mathbf{r}_{\|}, z, t\right)=Z \delta(x) \delta(y) \delta(z-v t), \\
& \rho_{\mathrm{ex}}\left(\mathbf{r}_{\|}, z, t\right)=Z \delta(x) \delta(y-v t) \delta\left(z-z_{0}\right) .
\end{aligned}
$$

Equations (10a) and (10b) represent the motion of the moving charge perpendicular to the graphene plane (piercing it) and the motion of the moving charge parallel to the graphene plane at a height $z=z_{0}$, respectively. The Fourier transform in $\mathbf{r}_{\|}$and $t$ of the charge distributions gives $\rho_{\mathrm{ex}}(\mathbf{k}, z, \omega)=$ $Z / v e^{i \omega z / v} \equiv Z / v e^{i z k_{z}}$ and $\rho_{\mathrm{ex}}(\mathbf{k}, z, \omega)=Z \delta\left(z-z_{0}\right) 2 \pi \delta(\omega-$ $k_{y} v$ ) for Eqs. (10a) and (10b), respectively. In both cases, we see a linear relation between the wave number and frequency: $k_{z}=\omega / v$ and $\omega=k_{y} v$ in the perpendicular and parallel motion, respectively. Note, however, that due to lack of translation invariance along the $z$ direction, $k_{z}$ is not a conserved quantity; this implies a nontrivial EEL spectrum. For ease of notation for later use, we now introduce a common prefactor $\Phi_{0} \equiv \frac{Z n_{0} e^{3}}{4 \epsilon_{0}^{2} m_{g}} \frac{1}{v^{2}}=\frac{Z e}{\epsilon_{0}} \alpha \frac{v_{F} c}{v^{2}} k_{F}$ that will serve to make many integrals dimensionless. Note that $\Phi_{0}$ has units of electric potential. Since $k_{F}=2 \pi / \lambda_{F}$, where $\lambda_{F}$ is the Fermi wavelength, $\Phi_{0}$ can be interpreted as the average Coulomb energy between two particles in the electron gas.

\section{A. Motion perpendicular to the graphene sheet}

An experimental method of accessing graphene surface plasmons is measuring the energy loss of an electron (or charged particle in general) when it passes through a graphene sheet. With this in mind we first consider the motion perpendicular to the graphene plane. The induced potential is given by

$$
\Phi_{\text {in }}(\mathbf{k}, z, \omega)=\Phi_{0} v^{2} \frac{e^{-k|z|}}{\omega^{2}-\omega_{\mathrm{spp}}^{2}} \frac{2 v k}{v^{2} k^{2}+\omega^{2}} .
$$

Fourier transforming to real space and time we have

$$
\Phi_{\mathrm{in}}\left(\mathbf{r}_{\|}, z, t\right)=\Phi_{0} \int v^{2} \frac{d \omega d \mathbf{k}}{(2 \pi)^{3}} \frac{e^{-k|z|} e^{i\left(\mathbf{k} \cdot \mathbf{r}_{\|}-\omega t\right)}}{(\omega+i \eta)^{2}-\omega_{\mathrm{spp}}^{2}} \frac{2 v k}{v^{2} k^{2}+\omega^{2}}
$$

where $\eta$ is a small positive real number added to account for causality. The angular integral gives $2 \pi J_{0}(k r)$. After performing the frequency integral we obtain

$$
\Phi_{\text {in }}(\mathbf{r}, z, t)=\Phi_{0} \int_{0}^{\infty} v^{3} \frac{k^{2} d k}{2 \pi} e^{-k|z|} J_{0}(k r) I_{1}\left(\omega_{\text {spp }}, k, t\right),
$$

where

$$
\begin{aligned}
I_{1}\left(\omega_{\mathrm{spp}}, k, t\right)= & -\theta(t) \frac{\sin \left(\omega_{\mathrm{spp}} t\right)}{\omega_{\mathrm{spp}}} \frac{1}{\omega_{\mathrm{spp}}^{2}+v^{2} k^{2}} \\
& -\frac{1}{2 v k} \frac{e^{-v k|t|}}{v^{2} k^{2}+\omega_{\mathrm{spp}}^{2}} .
\end{aligned}
$$

Therefore the problem of finding the induced electrostatic potential amounts to a simple quadrature. In Fig. 2, we represent $\Phi_{\text {in }}(r, 0, t)$ as a function of the distance to the origin for four different times. For shorter times we see the formation of the surface plasmon wave. At longer times, the surface plasmon has propagated a given distance. It is clear that the electrostatic disturbance is not monochromatic since a single wavelength cannot be identified from the figure. As we will see in the next section, this will translate into a nontrivial spectrum for the energy loss of a charged particle when it transverses a graphene sheet. 

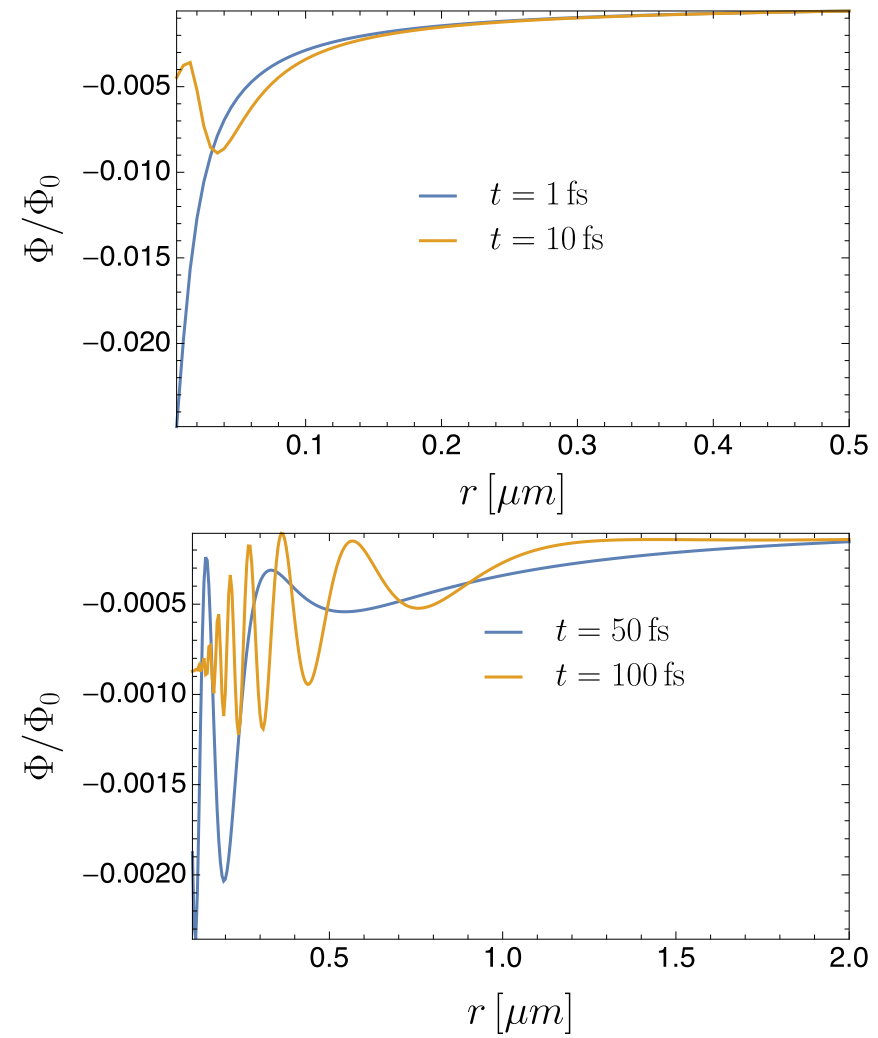

FIG. 2. Potential at position $z_{0}=0.1 \mu \mathrm{m}$ above the graphene sheet $\left(E_{F}=0.37 \mathrm{eV}\right)$ for $t=1,10 \mathrm{fs}$ (top) and $t=50,100 \mathrm{fs}$ (bottom) and for a particle of speed $v=0.01 c$. At large distances the induced potential approaches zero. At shorter times, we witness the formation of the surface plasmon polariton. At longer times the disturbance in the electronic density propagates way from the point $r=0$. The moving electric charge starts at the graphene sheet. As time evolves oscillatory behavior develops in the induced potential associated with the modulation of the electronic charge in the graphene sheet. The particle kinetic energy is about $25 \mathrm{eV}$. Note that the potential oscillations are not characterized by a well defined wavelength, meaning that surface plasmons of different wave numbers are excited simultaneously. The reader is invited to compare these plots with Fig. 2 of Ref. [32], which also considered the dynamics of graphene plasmons induced by swift electrons.

\section{B. The EEL spectrum}

Here we compute the electron-energy loss spectrum. This problem has been considered for graphene before using a completely different formalism [28]. Using the same methods of the previous reference, the problem of a $2 \mathrm{D}$ electron gas has also been considered [29-31], including the case where a magnetic field is present. Recently, the transient dynamics of 2D graphene-plasmons launched by swift electrons has also been considered [32].

To the goal in view, we need the quantity $E_{z}(0, z, \omega)=$ $-\partial \Phi_{\text {in }}(0, z, \omega) / \partial z$ since by definition the EEL spectrum reads [33]

$$
\Gamma(\omega)=\frac{Z e}{\pi \hbar \omega} \int_{-\infty}^{\infty} d t \Re\left\{e^{i \omega t} \mathbf{V} \cdot \mathbf{E}(0, v t, \omega)\right\},
$$

where $\mathbf{V}=(0,0, v)$ and $Z=1$ for the electron, and the symbol $\Re$ stands for the real part. The induced electrostatic potential

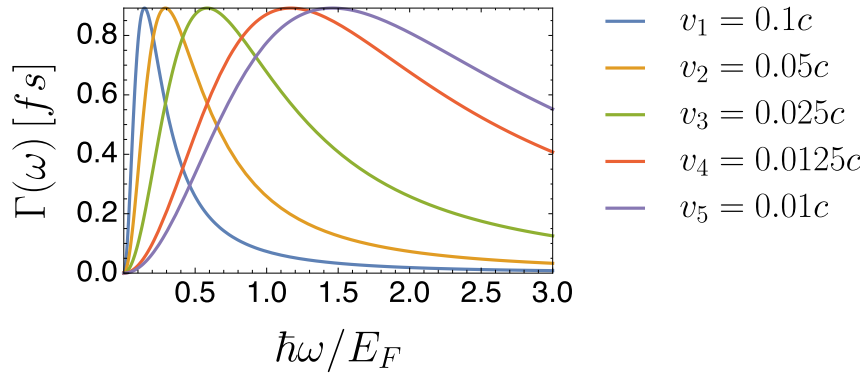

FIG. 3. Loss spectrum as a function of the energy for five speeds to the electron. The peaks disperse as a function of the speed of the electrons. This allows to retrieve the dispersion of the plasmons. The wave number $k_{\text {res }}$, which satisfies the condition $\omega_{\text {spp }}\left(k_{\text {res }}\right)=k_{\text {res }} v$, is connected to the resonance frequency $\omega_{\text {res }}$, the maximum of the EEL spectrum. This relation allows to reconstruct the surface plasmon dispersion from the EEL spectrum. The Fermi energy is $E_{F}=0.4 \mathrm{eV}$. The long tail as a function of frequency suggests that a continuum of surface plasmons is excited by the moving charge.

reads

$$
\Phi_{\text {in }}(0, z, \omega)=\Phi_{0} \int_{0}^{\infty} v^{2} \frac{k d k}{2 \pi} \frac{e^{-k|z|}}{(\omega+i \eta)^{2}-\omega_{\text {spp }}^{2}} \frac{2 v k}{v^{2} k^{2}+\omega^{2}} .
$$

Therefore it follows that the EEL spectrum can be written as

$$
\begin{aligned}
\Gamma(\omega)= & \frac{Z e}{\pi \hbar \omega} \Phi_{0} \int_{-\infty}^{\infty} d t \int_{0}^{\infty} v^{3} \frac{k^{2} d k}{2 \pi} \frac{2 v k}{v^{2} k^{2}+\omega^{2}} \\
& \times \frac{\operatorname{sgn}(t) e^{-k|v t|} e^{i \omega t}}{(\omega+i \eta)^{2}-\omega_{\text {spp }}^{2}},
\end{aligned}
$$

where the real part is implicit. Performing the time integral we find (using the Sokhotski-Plemelj theorem)

$$
\begin{aligned}
\Gamma(\omega)= & \frac{Z e}{\pi \hbar \omega} \Phi_{0} \int_{0}^{\infty} v^{3} \frac{k^{2} d k}{2 \pi} \frac{2 v k}{v^{2} k^{2}+\omega^{2}} \\
& \times \frac{2 \omega}{k^{2} v^{2}+\omega^{2}} \pi \delta\left(\omega-\omega_{\text {spp }}\right) .
\end{aligned}
$$

Writing $\omega_{\mathrm{spp}}=\sqrt{a k}$, where the parameter

$$
a=2 \alpha E_{F} c / \hbar
$$

has units of acceleration [see Eq. (B15)], we can easily integrate the delta function, reading

$$
\Gamma(\omega)=Z^{2} \frac{2 \hbar}{E_{F}} \frac{\omega^{2} v^{2} / a^{2}}{\left(\omega^{2} v^{2} / a^{2}+1\right)^{2}} .
$$

This result that has been obtained in the literature before [34] using a different method based on reflection coefficients. Equation (20) has a maximum at the frequency

$$
\hbar \omega_{\mathrm{res}} / E_{F}=2 \alpha \frac{c}{v}
$$

corresponding to an efficient excitation of surface plasmons of that frequency. We plot $\Gamma(\omega)$ in Fig. 3. From this figure, we see that the dispersion shifts towards higher energies as the speed of the moving electron decreases, in agreement with Eq. (21). Looking at the frequency where the EEL spectrum has a maximum, we can find the surface plasmon frequency. 
This frequency coincides with the interception of the $\omega_{\text {spp }}$ curve with the line $\omega=k_{z} v$, as can be seen in Fig. 4. This allows to retrieve the wave number $k_{z}$ of the surface plasmon associated with the $\omega_{\text {res }}$ obtained from the EEL spectrum. We note, however, that this process of exciting surface plasmons does not produce a monochromatic wave, as can be anticipated from the broadening of the EEL spectrum and from Figs. 2 and 4. Note that from the latter figure, we cannot attribute a single wavelength to the potential disturbance. As we will see, the motion of an electron parallel to a graphene sheet is able to induce a monochromatic plasmon. In this context, it is interesting to note that more than $50 \%$ of the energy of the surface plasmon-polariton is located in the graphene itself [35]. Therefore the incoming charged particle interacts strongly with the electronic disturbance in graphene.

\section{Motion parallel to the graphene sheet: Kelvin and Mach wakes}

We consider here the case of a charge moving parallel to a graphene sheet at a constant height $z_{0}$ (experimentally we could consider an EEL experiment with a tilted graphene sample). The calculation of the potential when the charge moves parallel to graphene follows from the Fourier representation of the charge density. In this case, the calculation is simpler due to the presence of two Dirac delta functions, but an integral in the coordinates $k_{x}$ and $k_{y}$ still remains. Let us compute the potential $\Phi_{\text {in }}(\mathbf{r}, z, t)$ starting from

$$
\Phi_{\text {in }}(\mathbf{k}, z, \omega)=2 \pi \Phi_{0} v^{2} \frac{e^{-k\left(|z|+z_{0}\right)}}{\omega^{2}-\omega_{\mathrm{spp}}^{2}} \delta\left(\omega-v k_{y}\right) .
$$

Note that the delta function implies that the particle disperses with a frequency given by $\omega=v k_{y}$.

Fourier transforming Eq. (22) to real space and time, we obtain in polar coordinates

$$
\begin{aligned}
\Phi_{\text {in }}\left(\mathbf{r}_{\|}, z, t\right)= & \Phi_{0} v^{2} \int_{0}^{2 \pi} \frac{d \theta}{2 \pi} \int_{0}^{\infty} \frac{k d k}{2 \pi} \\
& \times \frac{e^{-k\left(|z|+z_{0}\right)} e^{i k\left[r \cos \left(\theta-\theta^{\prime}\right)-v t \cos \theta\right]}}{(k \cos \theta v)^{2}+i \operatorname{sgn}(\cos \theta) \eta-a k}
\end{aligned}
$$

where $\theta^{\prime}$ is the polar angle of $\mathbf{r}_{\|}$. Using the Sokhotski-Plemelj theorem, it follows that $(\mathcal{f}$ stands for principal value of the integral)

$$
\begin{aligned}
\Phi_{\mathrm{in}}\left(\mathbf{r}_{\|}, z, t\right)= & \Phi_{0} \int_{0}^{2 \pi} \frac{d \theta}{2 \pi} \int_{0}^{\infty} \frac{d k}{2 \pi} \frac{e^{-k \beta} e^{i k \gamma}}{k \cos ^{2} \theta-a / v^{2}} \\
& -i \pi \Phi_{0} \int_{0}^{2 \pi} \frac{d \theta}{2 \pi} \int_{0}^{\infty} \frac{d k}{2 \pi} e^{-k \beta} e^{i k \gamma} \operatorname{sgn}(\cos \theta) \\
& \times \delta\left(k \cos ^{2} \theta-a / v^{2}\right) .
\end{aligned}
$$

Let us introduce the change of variables $k \cos ^{2} \theta-a / v^{2}=\kappa$, which modifies the integral to

$$
\begin{aligned}
\Phi_{\text {in }}\left(\mathbf{r}_{\|}, z, t\right)= & \Phi_{0} \int_{0}^{2 \pi} \frac{d \theta}{(2 \pi)^{2}} \frac{e^{-f(\theta)}}{\cos ^{2} \theta} f_{-a / v^{2}}^{\infty} d \kappa \frac{e^{-v^{2} f(\theta) \kappa / a}}{\kappa} \\
& -i \pi \Phi_{0} \int_{0}^{2 \pi} \frac{d \theta}{(2 \pi)^{2}} \frac{e^{-f(\theta)}}{\cos ^{2} \theta} \operatorname{sgn}(\cos \theta),
\end{aligned}
$$
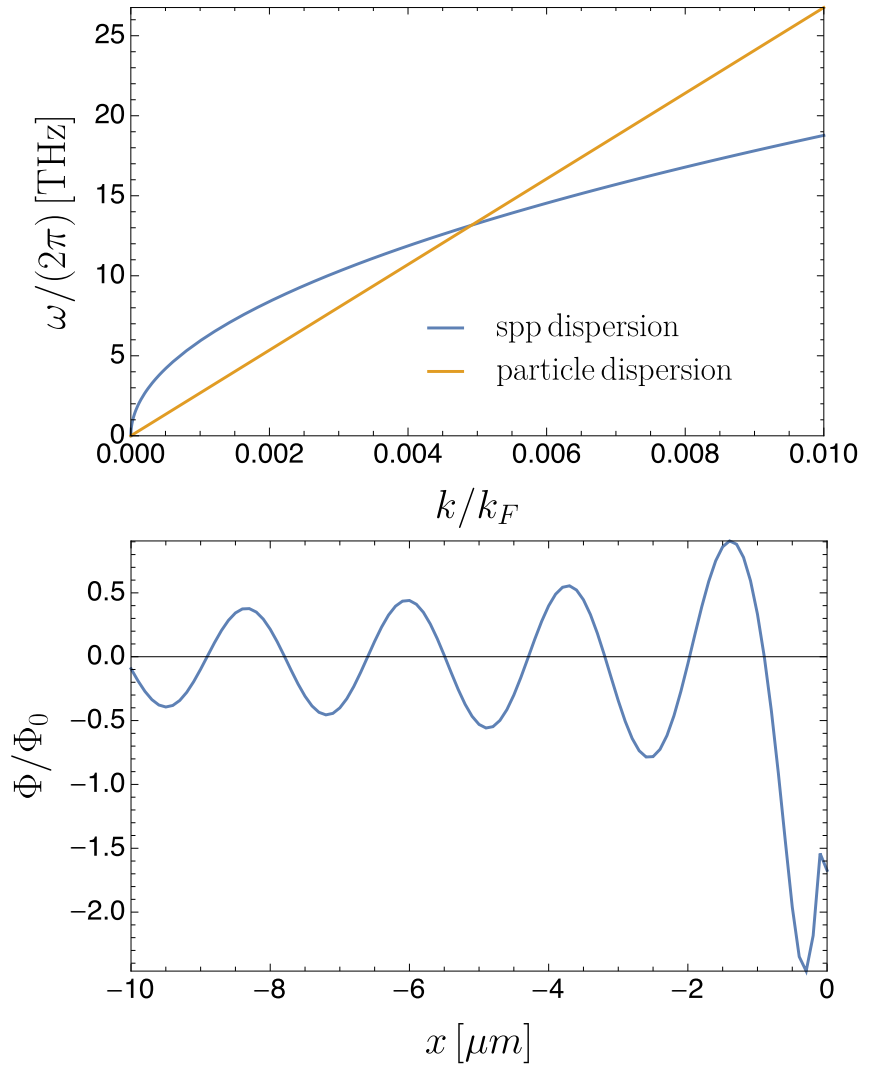

FIG. 4. Spectrum (top) and electrostatic potential (bottom) along the direction of propagation of the moving particle. The particle dispersion, given by $\omega=k v$, is represented on the left image by the orange line. It intercepts the SPP dispersion at $k / k_{F}=0.00495$, corresponding to a SPP wavelength of $\lambda_{\text {spp }}=2 \pi / k \approx 2.3 \mu \mathrm{m}$. This value matches well the distance between successive crests of the potential, $\Delta y \approx 2.4 \mu \mathrm{m}$. Changing the velocity of the particle changes the slope of the orange line and therefore the wavelength of the SPP, which turns into different distances between two successive crests of the potential. The parameters are $E_{F}=0.37 \mathrm{eV}$, corresponding to an electronic density equal to $10^{5} \mu \mathrm{m}^{-2}, v=0.1 c$, $z_{0}=0.01 \mu \mathrm{m}$, and $t=0 \mathrm{fs}$.

where $\beta=|z|+z_{0}, \gamma=\left[r \cos \left(\theta-\theta^{\prime}\right)-v t \cos \theta\right]$, and

$$
f(\theta)=\frac{a}{\cos ^{2} \theta v^{2}}[\beta-i \gamma(\theta)] .
$$

The principal value of the integral over $\kappa$ gives the exponential integral function, $\operatorname{Ei}(x)$, and the integral of the delta function is elementary. It then follows that

$$
\begin{aligned}
\Phi_{\text {in }}\left(\mathbf{r}_{\|}, z, t\right)= & -\Phi_{0} \int_{0}^{2 \pi} \frac{d \theta}{(2 \pi)^{2}} \frac{e^{-f(\theta)}}{\cos ^{2} \theta} \operatorname{Ei}[f(\theta)] \\
& -i \pi \Phi_{0} \int_{0}^{2 \pi} \frac{d \theta}{(2 \pi)^{2}} \frac{e^{-f(\theta)}}{\cos ^{2} \theta} \operatorname{sgn}(\cos \theta) .
\end{aligned}
$$

In Fig. 5, we show two examples of the electrostatic potential induced by the charged particle moving parallel to graphene at a distance $z_{0}$ from it. It is evident that the surface plasmons propagate in the form of ship wakes. Contrary to conventional wisdom, ship wakes are not [36] necessarily given by Kelvin theory and the same happens for plasmonic wakes induced by the moving charge, as we shall see. 

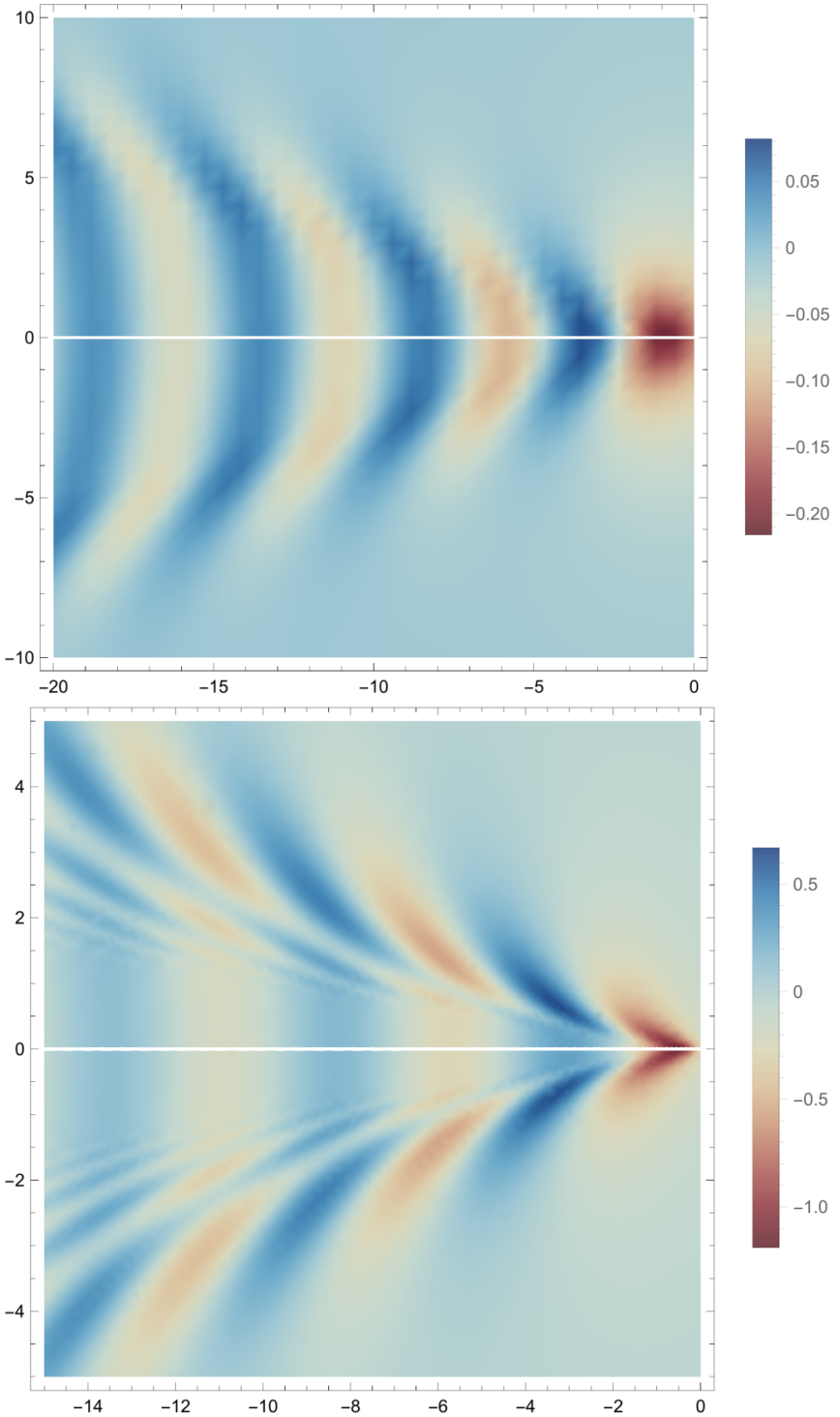

FIG. 5. Electrostatic potential, in units of $\Phi_{0}$, in graphene for a particle moving parallel to it at $z_{0}=1 \mu \mathrm{m}$ with speed $v=0.1 \mathrm{c}$ (top panel; $E_{F}=0.17 \mathrm{eV}$ ) and at $z_{0}=0.1 \mu \mathrm{m}$ with speed $v=0.1 c$ (bottom panel; $E_{F}=0.17 \mathrm{eV}$ ). The axes of the figures are in $\mu \mathrm{m}$. A Kelvin wake develops on the graphene sheet in the image of the top panel. Indeed, in the top panel the half-opening angle is about $\sim(22.8 \pm 0.3)$ o, whereas in the bottom one is about $\sim(16.8 \pm 0.3)^{\circ}$. Note that the electrostatic potential fluctuations are much larger for the case depicted in the bottom panel, due to a closer proximity of the moving charge to graphene. Also note the presence of a plane wave in the central region of the wake (more evident in the bottom panel). (The horizontal and vertical scales in the two panels are different.)

According to Kelvin theory, half of the angle of the cone of a ship wake is given by

$$
\theta_{K}=\arctan \frac{1}{\sqrt{8}} \approx 19.47^{\circ} .
$$

In other words, it is a constant number independent of the speed of the ship. For future notice, it is important to clarify how the opening angle of the plasmon wake is determined from our simulations. For a fixed value of $|\mathbf{r}|$, we first compute from the electrostatic potential the three components of the electric field as a function of the angle $\theta^{\prime}$. Next, we then use these results to compute the absolute value of the electric field and fit a Gaussian of the form $A e^{-\left(\theta^{\prime}-\theta_{\max }\right)^{2} / \sigma}$, where $\theta_{\max }$ is the angle where the intensity of the electric field is maximum, and $A$ and $\sigma$ are fitting parameters. The opening half angle is then defined as the angle where the fitting function has the value of 0.61 of its maximum, at a radial position value given by $|\mathbf{r}|=(2+1 / 4) 2 \pi v^{2} / a$ (other choices of $|\mathbf{r}|$ lead to the same results). Using this procedure, and with the help of dimensional analysis, we have found that half the aperture of the cone is given with good accuracy by (in degrees)

$$
\theta^{\prime} \approx \frac{1}{\delta} \sqrt{\frac{z_{0} a}{v^{2}}} \equiv \frac{1}{\delta} \frac{1}{\mathrm{Fr}_{\mathrm{pl}}}
$$

for $\mathrm{Fr}_{\mathrm{pl}} \gg 1$, where $\delta$ is a real constant that we have found to be of the order of $\delta \approx 0.019 \pm 0.001$ ( $\delta \approx 1.09$ for $\theta^{\prime}$ in radians) and $\mathrm{Fr}_{\mathrm{pl}}$ is the plasmonic Froude number (see discussion in Sec. V). We note that formula (29) should work well only in the large Froude number regime and should be understood as the first term in powers of $1 / \mathrm{Fr}_{\mathrm{pl}}$ of a more complex expression. From the numerical data, we have identified, a transition from a Mach-type wavefront, where the opening angle of the plasmon wake follows the law $\theta \propto 1 / v$, at high speeds (for the remaining parameters fixed) to a Kelvin-type one, where the angle of the plasmon wake is independent of the speed of the moving charge (see discussion ahead). Indeed, for slow speeds compared to $c$ (and small Froude numbers) the wavefront is always Kelvin-like, that is, with an opening angle for the wake independent of the speed of the moving charge.

A study of the evolution of the plasmon wake from Kelvintype to Mach-type is given in Fig. 6. Note the transition located at $\mathrm{Fr}_{\mathrm{pl}} \sim 2$ from a Mach-type wake to a Kelvin-type one, as the Froude number decreases. The existence of such transition was first pointed out by Shi et al. [37], who solved an identical problem numerically, but without offering an interpretation to the phenomenon as they were unable to identify the Froude number for graphene. A study of the prediction given by Eq. (29) and the estimation based on the procedure described above is given in Table I. The agreement between the numbers in the two rows is good for values of $\mathrm{Fr}_{\mathrm{pl}}$ larger than 2, showing that the ansatz $\theta=1 /\left(\delta \mathrm{Fr}_{\mathrm{pl}}\right)$ does a good job at predicting the values obtained from the calculation of the absolute value of the electric field. The last row gives the Froude number. We have, therefore, gathered evidence for the existence of a transition from Mach-type to Kelvin-type waves at a critical Froude number of $\mathrm{Fr}_{\mathrm{pl}}^{\mathrm{c}} \gtrsim 2$ (note that the ratio $v / c$ is not the appropriate quantity to analyze this problem).

For the parameters considered in Fig. 6, and as noted above, we have $\mathrm{Fr}_{\mathrm{pl}} \approx 2.3$ for $v / c=0.25$, the speed at which the transition from the Mach-like to Kelvin-like regime occurs (for the given parameters). Therefore the transition between the two regimes is controlled by Froude number, with the transition occurring roughly for $\mathrm{Fr}_{\mathrm{pl}} \approx 2$. This result should be a generic feature of plasmonic wakes in graphene. Finally, we note that the region of Kelvin-type wakes has a constant angle (gray dashed line in Fig. 6) of $(21.4 \pm 1.4)^{\circ}$, a value larger than that predicted by Kelvin's theory, but with Kelvin's value within the interval of uncertainty. This larger value of the opening angle happens due to the definition we have used for determining it. 
TABLE I. Estimation of half the angle $\theta_{\text {est }}$ of the cone associated with the moving plasmons induced by a moving charge compared with the prediction of equation $\theta^{\prime}=1 /\left(\delta \mathrm{Fr}_{\mathrm{pl}}\right)$, with $\delta \approx 0.019$. Note that the validity of this formula is restricted to $\mathrm{Fr}_{\mathrm{pl}}>2$. The parameters are $E_{F}=0.17 \mathrm{eV}$ and $z_{0}=1 \mu \mathrm{m}$. Figure 6 plots the numbers of the table in a log-log scale. Also note the transition at about $v / c \approx 0.25$. (See text for the method used to estimate $\theta_{\text {est }}$.) The last line gives the plasmonic Froude number (see discussion in $\left.\operatorname{Sec} . \mathrm{V}\right), \mathrm{Fr}_{\mathrm{pl}}=\sqrt{v^{2} /\left(z_{0} a\right)}$. It is clear that the criterion for the validity of formula (29) is related to the value of the Froude number: when Fr $\mathrm{p}_{\mathrm{pl}} \gtrsim 2$ the formula works well.

\begin{tabular}{|c|c|c|c|c|c|c|c|c|c|c|c|c|c|c|}
\hline$v / c$ & 0.075 & 0.1 & 0.125 & 0.15 & 0.2 & 0.25 & 0.3 & 0.35 & 0.4 & 0.5 & 0.6 & 0.7 & 0.8 & 0.95 \\
\hline$\theta_{\text {est }}$ & 22.8 & 22.8 & 21.6 & 21.6 & 20.4 & 19.2 & 18 & 16.8 & 14.4 & 12 & 9.6 & 8.4 & 7.2 & 6 \\
\hline$\theta^{\prime}$ & 74 & 56 & 44 & 37 & 28 & 22.2 & 18.5 & 15.9 & 13.9 & 11.1 & 9.3 & 7.9 & 6.9 & 5.8 \\
\hline $\mathrm{Fr}_{\mathrm{pl}}$ & 0.68 & 0.91 & 1.1 & 1.4 & 1.8 & 2.3 & 2.7 & 3.2 & 3.6 & 4.5 & 5.4 & 6.3 & 7.2 & 8.6 \\
\hline
\end{tabular}

Indeed, adopting a slightly different criterion, the angle values would be slightly different, but the transition would occur at the same Froude number (results not shown). That is, the existence of a transition from Mach-like to Kelvin-like behavior does not depend on the criterion used to define the opening angle of the cone. We shall discuss more on all the above in Secs. IIID and $\mathrm{V}$.

We can also compute the power added to the charged particle using the expression $\mathcal{P}=Z e \mathbf{V} \cdot \mathbf{E}\left(0, v t, z_{0}, t\right)$, where $\mathbf{V}=(0, v, 0)$ and $\mathbf{E}=\mathbf{E}(x, y, z, t)$. This means that we need to compute the $y$ component of the field, which follows from $E_{y}=-\partial \Phi_{\text {in }}\left(\mathbf{r}_{\|}, z, t\right) / \partial y$. Once the electric field is known, the power can be determined. The calculation is consistent if the energy loss is small compared to the kinetic energy of the particle. Since the field $E_{y}\left(0, v t, z_{0}, t\right)$ is time independent, the power loss is also time independent. We have verified in our numerics (results not shown) that the energy loss is of the order of $2 \mathrm{meV}$, for a particle with speed $v=0.1 c$ (kinetic energy $2.5 \mathrm{keV}$ ) propagating over graphene for a distance of $3 \mu \mathrm{m}$. We note that the situation is different for the motion of a charged particle perpendicular to the graphene plane due to lack of translation invariance. In this latter case, the power loss is time dependent.

Although numerical calculations are a powerful way to accumulate understanding about a complex problem, much insight can be gained from deriving analytical results, even when they are only valid in special limits. In the present section, we have conducted a fully numerical analysis of the plasmonic wake. In the next section, two special limits are considered where it became possible to obtain closed analytical expressions for the shape of the wake.

\section{Approximate analytical formulas for the plasmonic wake}

In this section, we derive analytical formulas for the plasmonic wakes valid in the limits $a z_{0} / v^{2} \gg 1$ and $a z_{0} / v^{2} \ll 1$ (a similar problem has been considered in the past for a charge moving near a metal-vacuum interface [38]). The first limit corresponds approximately to the case of the top panel of Fig. $5\left(a z_{0} / v^{2} \simeq 1.2\right)$, whereas the second limit corresponds to the bottom panel of the same figure $\left(a z_{0} / v^{2} \simeq 0.12\right)$. The exact form of the plasmonic wake is given by Eq. (27). However, we are interested here in obtaining approximate analytical expressions for the wake, which can then be used to gain some insight on its properties. To this end, we consider the asymptotic expression for the $\operatorname{Ei}(z)$ function, which to leading order reads $\operatorname{Ei}(z) \sim e^{z} / z$ $(\Re z>0)$. This shows that to leading order the first integral in
Eq. (27) is elementary and does not contribute significantly to the form of the wake, as this comes from the exponential in the second integral [a careful numerical study of both integrals in Eq. (27) shows that this statement is only approximately correct in some regimes]. In order to derive the needed asymptotic expressions, we note that we need to compute the real part of the following integral [ignoring, for the time being, contributions coming from the first integral in Eq. (27)],

$$
I_{2}=-i \pi \int_{0}^{2 \pi} d \theta \frac{e^{-\frac{a z_{0}}{v^{2} \cos ^{2} \theta} e^{\frac{i a r \cos \left(\theta-\theta^{\prime}\right)}{v^{2} \cos ^{2} \theta}}}}{\cos ^{2} \theta} \operatorname{sgn}(\cos \theta),
$$

which can be shown to equal

$$
\begin{aligned}
\Re I_{2}= & 2 \pi \int_{-\pi / 2}^{\pi / 2} d \theta \frac{e^{-\frac{a z_{0}}{v^{2} \cos ^{2} \theta}}}{\cos ^{2} \theta} \sin \left(\frac{\operatorname{ar}}{v^{2}} \frac{\cos \theta^{\prime}}{\cos \theta}\right) \\
& \times \cos \left(\frac{\operatorname{ar}}{v^{2}} \frac{\tan \theta}{\cos \theta} \sin \theta^{\prime}\right) .
\end{aligned}
$$

We now introduce the change of variable $u=\tan \theta$. This implies that $1 / \cos ^{2} \theta=1+u^{2}$ and $d u=\sec ^{2} \theta d \theta$. Therefore the integral reads

$$
\begin{aligned}
\Re I_{2}= & 2 \pi e^{-a z_{0} / v^{2}} \int_{-\infty}^{\infty} d u e^{-u^{2} a z_{0} / v^{2}} \sin \left(\frac{a r}{v^{2}} \cos \theta^{\prime} \sqrt{u^{2}+1}\right) \\
& \times \cos \left(\frac{a r}{v^{2}} \sin \theta^{\prime} u \sqrt{u^{2}+1}\right) .
\end{aligned}
$$

Next, we observe that for $a z_{0} / v^{2} \gg 1$ the kernel of the integral is strongly peaked at $u=0$, due to the Gaussian exponential. Therefore, in this regime, we introduce the approximation

$$
\begin{aligned}
\Re I_{2} \approx & 2 \pi e^{-a z_{0} / v^{2}} \int_{-\infty}^{\infty} d u e^{-u^{2} a z_{0} / v^{2}} \cos \left(\frac{a r}{v^{2}} \sin \theta^{\prime} u\right) \\
& \times \sin \left(\frac{a r}{v^{2}} \cos \theta^{\prime}\left(1+u^{2} / 2\right)\right),
\end{aligned}
$$

which, applying the exponential representation of the trigonometric formulas, can be seen as a Gaussian integral, which has the elementary solution

$$
\Re I_{2} \approx-4 \pi^{3 / 2} e^{-\beta} \frac{\Im\left(\sqrt{2 \beta-i \gamma} e^{-i \gamma-\frac{\lambda^{2}}{4 \beta+2 i \gamma}}\right)}{\sqrt{8 \beta^{2}+2 \gamma^{2}}},
$$

where

$$
\begin{aligned}
& \beta=\frac{a z_{0}}{v^{2}}, \\
& \gamma=\frac{a r}{v^{2}} \cos \theta^{\prime}, \\
& \lambda=\frac{a r}{v^{2}} \sin \theta^{\prime} .
\end{aligned}
$$



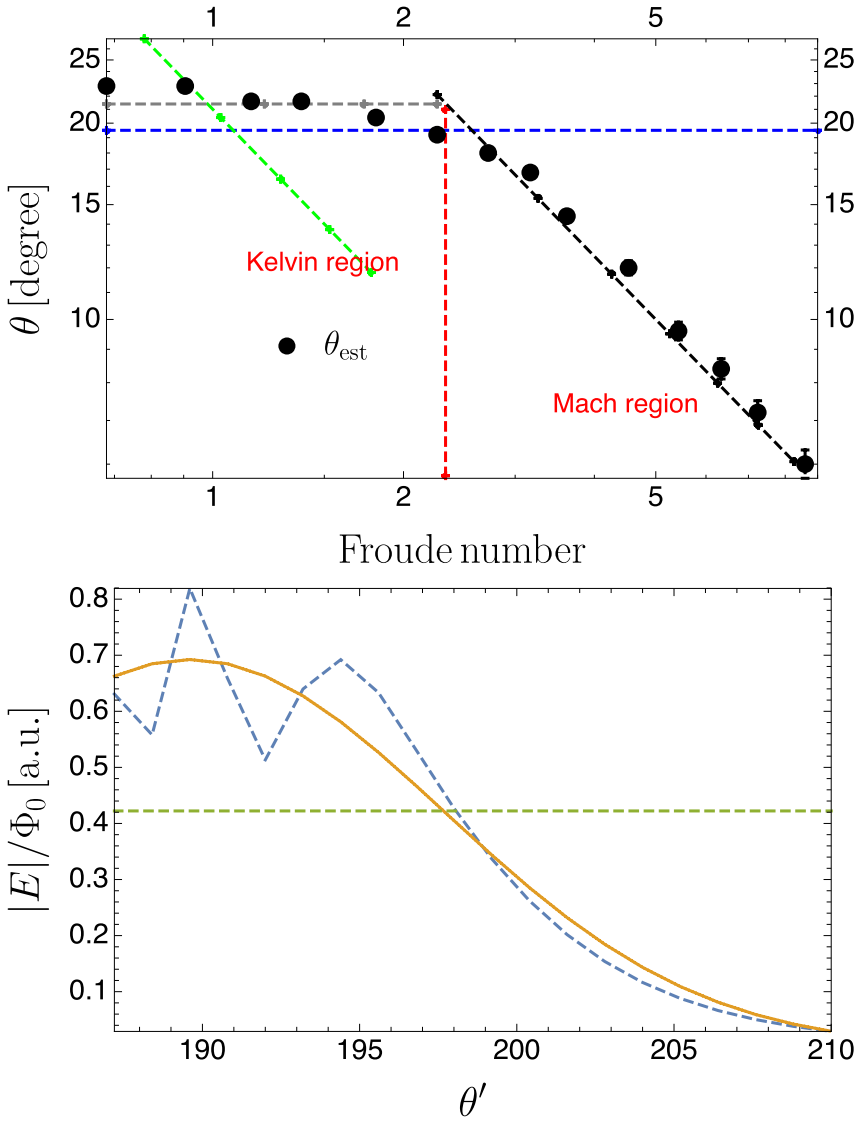

FIG. 6. Graphical representation of the data of Table I. (Top) Note the transition at the Froude number $\mathrm{Fr}_{\mathrm{pl}} \sim 2$ from Mach-type to Kelvin-type of wake, as the Froude number decreases. The parameters are $z_{0}=1 \mu \mathrm{m}$ and $E_{F}=0.17 \mathrm{eV}$, and the angle was measured at a distance $|\mathbf{r}|=(2+1 / 4) 2 \pi v^{2} / a$ (corresponding to two wavelengths plus one quarter) from the apex of the cone. The error of the data points was estimated to be $0.3^{\circ}$. Different parameters will give similar curves to this one. The angle $\theta_{\text {est }}$ is estimated from the electric field, for a given $v / c$ ratio, as explained in the text. The dashed black line represents the angle given by the formula $\theta=1 /\left(\delta \mathrm{Fr}_{\mathrm{pl}}\right)$ as function of the Froude number (this formula only holds in the Mach-type region of the Froude number). The horizontal dashed blue line represents Kelvin's result (see Sec. V for a more thorough discussion of the role of the Froude number). The horizontal gray line is a fit to the simulation data points in the Kelvin region (the transition from Machlike to Kelvin-like behavior is clearly seen to occur at the interception of the gray and black dashed curves). The green dashed line is an attempt to fit the simulation data points in Kelvin's region with the same expression used in the Mach region (this clearly fails to account for the behavior of the data). (Bottom) Here we give an example of the fitting procedure (see text for details). The dashed blue line is the simulation data, the orange solid line is the fit of the data to a Gaussian, and the dashed green line signals the value of 0.61 of the maximum of the fitting function which approximately corresponds to the inflection point of the curve $\left(0.61 \approx e^{-1 / 2}\right)$. The interception of the horizontal line with the solid curve defines the half angle of aperture of the wake cone. The speed of the particle is $v=0.1 c, E_{F}=0.17 \mathrm{eV}$, and $z_{0}=0.1 \mu \mathrm{m}$, corresponding to a Froude number of $\mathrm{Fr}_{\mathrm{pl}}=2.86$. In this example, we have $\theta_{\text {est }} \approx 196.8^{\circ}-180^{\circ}=16.8^{\circ}$.

For obtaining the second term in Eq. (27) we have to divide Eq. (34) by $4 \pi^{2}$. The obtained expression is valid for arbitrary large values of $r$ and describes qualitatively the formation of the wake due to the moving charge in its regime of validity. As noted above, the first integral in Eq. (27) contributes little to leading order in the form of the asymptotic expression of the exponential integral function. However, the regime $a z_{0} / v^{2} \gg 1$ is likely to be experimentally challenging to access (see discussion below). Therefore we would like to have an equation holding in the regime $a z_{0} / v^{2} \gtrsim 1$. Fortunately, this can be obtained treating the first integral approximately. The procedure is similar to that described above, except that in the end we still have to evaluate the additional integral coming from the principal value of the integral in the variable $\kappa$. The final result to the first integral in Eq. (27) reads (up to lowest order in the expansion of the arguments of the trigonometric functions)

$$
I_{1} \approx \Re\left[\frac{\sqrt{2}(-2 \beta+i \gamma)}{\pi \sqrt{(2 \beta-i \gamma)^{2}(2 \beta+i \gamma)}} D(s)\right],
$$

where $s=\sqrt{i \phi+\psi}$ and $D(z)$ is the Dawson integral, $D(z)=$ $e^{-z^{2}} \int_{0}^{z} e^{y^{2}} d y$, and

$$
\begin{aligned}
& \psi=\beta+\frac{4 \beta \lambda^{2}}{16 \beta^{2}+4 \gamma^{2}}, \\
& \phi=\frac{\gamma\left(8 \beta^{2}+2 \gamma^{2}-\lambda^{2}\right)}{2\left(4 \beta^{2}+\gamma^{2}\right)} .
\end{aligned}
$$

Therefore the electrostatic potential (27) is approximately given by the sum: $I_{1}+(2 \pi)^{-2} \mathfrak{R} I_{2}$. This result is in quantitative agreement with the exact fully numerical calculation of equation (27). A better analytical approximation than Eq. (36) to the first integral in Eq. (27) can be obtained, but the resulting expression is too cumbersome to be given here.

Let us next consider the regime $a z_{0} / v^{2} \ll 1$. In this case, the integral is dominated by values of $u$ in a large range centered at $u=0$. Therefore the arguments of the trigonometric functions are expanded differently than before as

$$
\begin{aligned}
\Re I_{2} \approx & 2 \pi e^{-a z_{0} / v^{2}} \int_{-\infty}^{\infty} d u e^{-u^{2} a z_{0} / v^{2}} \sin \left(\frac{a r}{v^{2}} \cos \theta^{\prime}|u|\right) \\
& \times \cos \left(\frac{a r}{v^{2}} \sin \theta^{\prime}\left(u^{2}+1 / 2\right)\right) .
\end{aligned}
$$

The integral can be expressed in terms of the error function, $\operatorname{erf}(x)$, as

$$
\Re I_{2} \approx-4 \pi^{3 / 2} e^{-\beta} e^{-\frac{\beta \gamma^{2}}{2\left(\beta^{2}+\lambda^{2}\right)}} \Re\left[\frac{e^{\frac{\gamma^{2}}{4 \beta+4 i \lambda}+i \lambda / 2} \operatorname{erf}\left(\frac{\gamma}{2 \sqrt{-\beta+\mathrm{i} \lambda}}\right)}{\sqrt{-\beta+i \lambda}}\right] .
$$

Again, the previous expression is valid for an arbitrary large $r$. It is interesting to note that the error function often appears in diffusion problems. Whether the propagation of the surface plasmons in this regime can be seen as a diffusion problem requires more work. Also in this case, we can obtain an expression for the first integral in Eq. (27). Proceeding as briefly described in the regime $a z_{0} / v^{2} \gg 1$, the expression for 

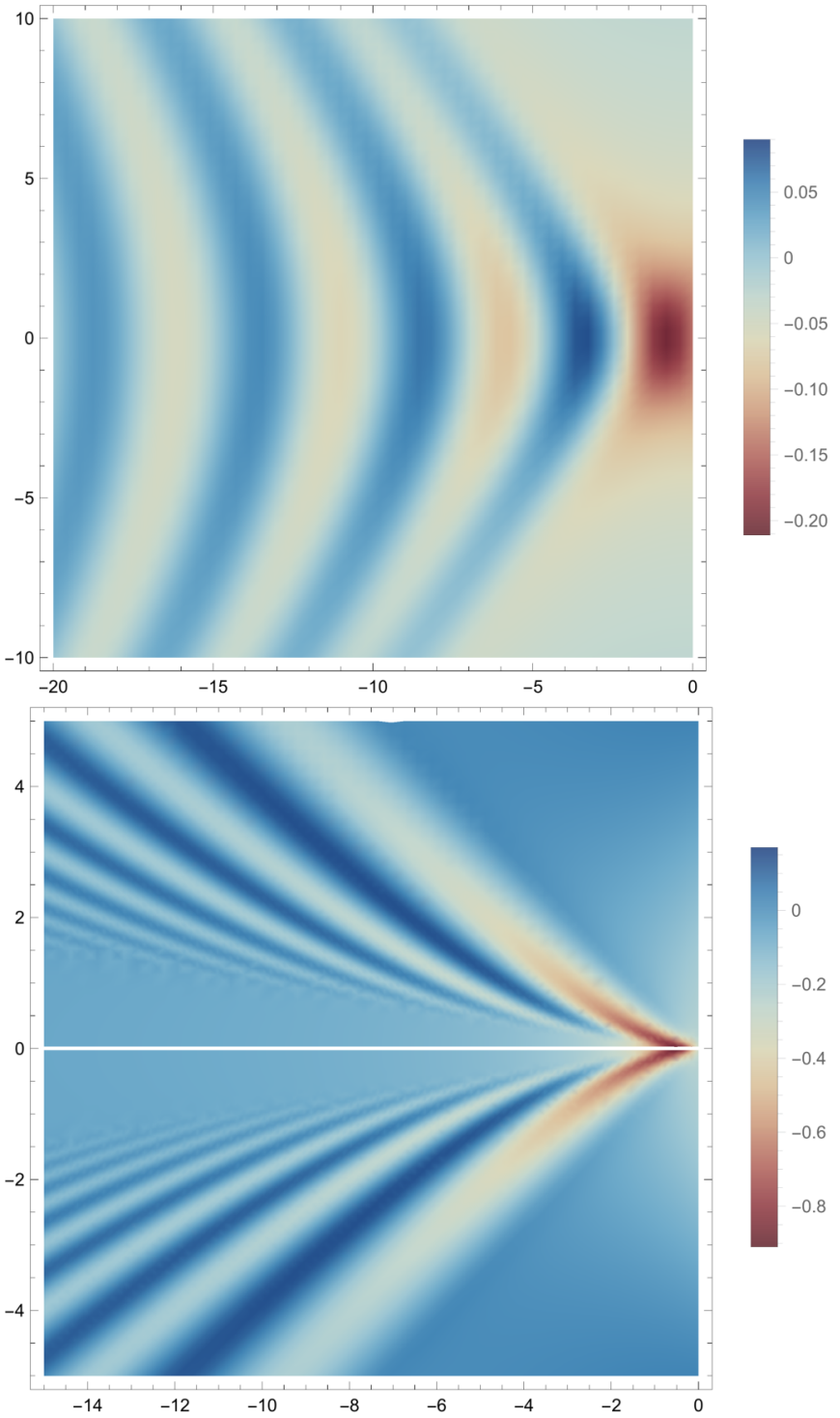

FIG. 7. Electrostatic potential, in units of $\Phi_{0}$, in graphene for a particle moving parallel to it at $z_{0}=1 \mu \mathrm{m}$ with speed $v=$ $0.1 c$ (top panel; $E_{F}=0.17 \mathrm{eV}$ ) and computed using equations (34) and (36), and at $z_{0}=0.1 \mu \mathrm{m}$ with speed $v=0.1 c$ (bottom panel; $E_{F}=0.17 \mathrm{eV}$ ) and computed using equations (39) and (40). Note the quantitative agreement between corresponding panels in this figure and Fig. 5. The axes of the figures are in $\mu \mathrm{m}$. The electrostatic potential was computed using the formula $\Phi_{\text {in }} / \Phi_{0}=$ $I_{1}+\Re I_{2} /\left(4 \pi^{2}\right)$

$I_{1}$ reads

$$
I_{1} \approx-\frac{\Re\left[\sqrt{\beta-i \lambda} e^{-s} \operatorname{erfi}(s)\right]}{\pi^{1 / 2} \sqrt{\beta^{2}+\lambda^{2}}}
$$

where in this case $s=\left(4 \beta^{2}+\gamma^{2}+6 i \beta \lambda-2 \lambda^{2}\right) /(4 \beta+4 i \lambda)$ and $\operatorname{erfi}(z)$ is the complex error function. As before, the electrostatic potential (27) is approximately given by the sum $I_{1}+(2 \pi)^{-2} \Re I_{2}$.

To compare the asymptotic expressions to the exact results we depict in Fig. 7, the same wakes shown in Fig. 5 but computed using Eqs. (34) and (36), and Eqs. (39) and (40).
It is clear that the shape of the approximated wakes given in Fig. 7 is in qualitative agreement with the wakes depicted in Fig. 5. The agreement between the wake for $z_{0}=1 \mu \mathrm{m}$ is only qualitatively accurate since the ratio $a z_{0} / v^{2} \simeq 1.2$ is not in the regime $a z_{0} / v^{2} \gg 1$. Had we chosen a larger $z_{0}$, or a smaller $v$, the agreement between the two wakes would have been quantitatively better. In particular, we note the disagreement in the value of the opening angle of the wake's cone (larger in the approximate wake). In the case of the bottom panel of Fig. 7, the agreement with the bottom panel of Fig. 5 is quite good, since in this case the wake for $z_{0}=0.1 \mu \mathrm{m}$ is more accurate, given that the ratio $a z_{0} / v^{2} \simeq 0.12$ can be considered to fulfill the condition $a z_{0} / v^{2} \ll 1$. Indeed, in both cases, the cone of the wake has the same coordinate $y \approx \pm 2 \mu \mathrm{m}$, for $x=-15 \mu \mathrm{m}$. It is also clear that the form of the wake in this regime differs considerably from the previous one. This is a striking manifestation of the two aforementioned regimes. The existence of these two regimes was put in evidence in the numerical studies of Table I and Fig. 6.

It is clear from this analytical analysis that there is a transition in the shape of the wake around $a z_{0} / v^{2} \sim 1$, which is precisely the parameter that enters in the formula (29), proposed based on intuition, numerical, and dimensional analysis. Therefore the regime $a z_{0} / v^{2} \gg 1$ defines the Kelvinlike behavior of the wake, whereas the opposite regime defines the Mach-like behavior.

If we zoom out the wake in the top panels of Figs. 5 and 7, we can clearly identify the presence of a plane wave superimposed on the wake. This is also evident in the bottom panel of Fig. 5 . This plane wave exhibits a number of crests and valleys. We note that the number of nodes and crests in the approximated wakes coincide with the same quantity in the exact wakes. Indeed if in Eq. (33), we make the approximation (since in this case the integral is dominated by values of $u \approx 0$ )

$$
\sin \left(\frac{a r}{v^{2}} \cos \theta^{\prime}\left(1+u^{2} / 2\right)\right) \approx \sin \left(\frac{a r}{v^{2}} \cos \theta^{\prime}\right),
$$

it is then clear that we have superimposed to the wake pattern a plane wave of the form $\sin \left(\operatorname{arcos} \theta^{\prime} / v^{2}\right)=\sin \left(a x / v^{2}\right)$. Considering the case of the top panel of Fig. 7, we have the ratio $a / v^{2} \approx 1.2 \mu \mathrm{m}^{-1}$. Therefore the wavelength of the wave reads $\lambda \approx 2 \pi v^{2} / a \approx 5 \mu \mathrm{m}$, meaning that within a propagation distance $\Delta x=20 \mu \mathrm{m}$ we should have four crests, which is exactly what is seen in the top panels of Figs. 5 and 7. The number of crests and valleys seen in the bottom panel of Fig. 5 is three, since $\Delta x=15 \mu \mathrm{m}$. We note the absence of the plane wave pattern in the wake of the bottom panel of Fig. 7; this is a consequence of the approximation of the argument of the sine-function used in the limit $a z_{0} / v^{2} \ll 1$ (we have used a large $u$ expansion and the plane wave depends on the small $u$ values, as seen in the previous equation).

In Fig. 8, we show the electrostatic potential, $\Phi / \Phi_{0}$, along the direction $\theta^{\prime}=\pi(y=0)$. The agreement between the exact and the approximated formulas is excellent. Note, in the central panel, the missing plane-wave oscillations in the approximated result for large Froude number; the reason for this has been discussed already. However, if we decrease $z_{0}$ for increasing the Froude number, the wavelength of the plane wave becomes very large and agreement between the approximated and numerically exact solutions is excellent. The agreement between 

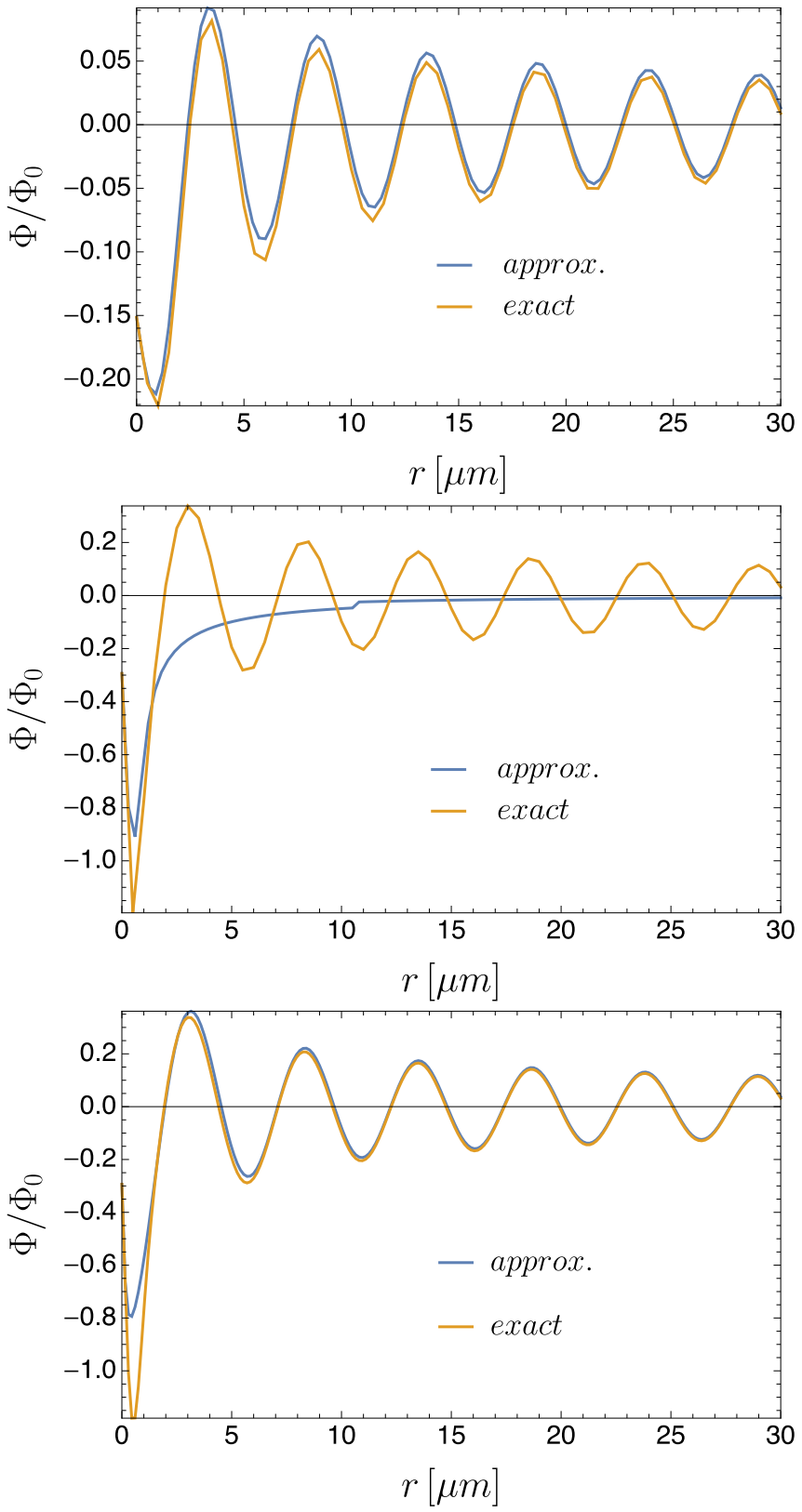

FIG. 8. Comparison of the exact and approximated expression for the potential, in units of $\Phi_{0}$, along the direction $\theta^{\prime}=\pi$. The parameters are the same as in Fig. 7. In the top panel, the Froude number is $\mathrm{Fr}_{\mathrm{pl}}=0.90$ and in the central and bottom ones is $\mathrm{Fr}_{\mathrm{pl}}=$ 2.86. Note the excellent agreement of the approximated result in the top panel, even though the Froude number is only slightly smaller than 1. Also note that in the central panel, for moderate Froude number, the plane wave is missing in the approximated curve [computed using Eqs. (39) and (40)]; the reason for this is discussed in the text. In the bottom panel, we improved over the approximation of the central panel using Eqs. (34) and (42). However, this level of approximation is only valid in the regime $a z_{0} / v^{2} \lesssim 1$ where the plane wave is well developed. All the approximated curves were computed using the formula $\Phi_{\text {in }} / \Phi_{0}=I_{1}+\Re I_{2} /\left(4 \pi^{2}\right)$.

the approximated curves and the numerical exact ones is also good for values of $y \neq 0$ (results not shown). The fact that the plane wave is missing in the limit of large Froude numbers hints that the approximation of the arguments of the trigonometric functions by their values in the limit $u \rightarrow \infty$ is too drastic. This conclusion suggests keeping for the value of $\Re I_{2}$ the same dependence that is used in the limit of small Froude number, since this part contains the plane wave, that is, we use Eq. (34) for $\Re I_{2}$. As for the integral $I_{1}$ we expand the arguments of the two cosines differently: the one responsible for the plane wave is expanded in the limit of small $u$, whereas the other is expanded in the limit of large $u$. With this procedure, we obtain for $I_{1}$ the result

$$
\begin{aligned}
I_{1} \approx & \frac{\sqrt{\pi / 2}}{4 \pi^{2}}\left[\frac{e^{-s_{1} / 2}}{\sqrt{2 \beta-i(\gamma-2 \lambda)}} f\left(s_{1} / 2\right)+\text { c.c. }\right] \\
& +\frac{\sqrt{\pi / 2}}{4 \pi^{2}}\left[\frac{e^{-s_{2} / 2}}{\sqrt{2 \beta-i(\gamma+2 \lambda)}} f\left(s_{2} / 2\right)+\text { c.c. }\right]
\end{aligned}
$$

with $s_{1}=2 \beta-2 i \gamma+i \lambda, s_{2}=2 \beta-i(2 \gamma+\lambda)$, and

$$
f(z)=i \pi \operatorname{erf}\left[i \sqrt{|z|} e^{i \arg (z) / 2}\right]
$$

where $\arg (z)$ is the argument of the complex number $z$ and $\Re z>0$. This last result for $I_{1}$ together with Eq. (34) prove to be accurate in the regime $a z_{0} / v^{2} \lesssim 1$, as seen in the bottom panel of Fig. 8. Therefore this approach includes the plane wave present in the wake at intermediated Froude numbers. However, being exceptionally good in describing the $\theta^{\prime}=\pi$ case, this approximation does not excel for moderate to large angular deviations from $\theta^{\prime}=\pi$, as it underestimates the amplitude of the plane wave along these directions.

From the previous analytical study, we learn that the plane wave existing in the wake has its wavelength controlled by the value of $v^{2} / a$ and while $z_{0}$ plays no immediate role in this. From the analytical solution we also learn that the effect of the moving charge on the plasmonic wake diminishes exponentially with $z_{0}$ due to the exponential factor $e^{-a z_{0} / v^{2}}$. Therefore the regime $a z_{0} / v^{2} \gg 1$ is likely to be experimentally challenging as the charge fluctuations are exponentially suppressed. This is also clear from the vertical scales of Fig. 7. From the previous discussion, it is obvious that the dimensionless ratio $z_{0} a / v^{2}$ plays a fundamental role in determining the nature of the wake. As argued in Sec. V this quantity is related to the Froude number of plasmonic wakes induced by the Coulomb dragging effect of the passing charge.

Equation (39) can also be used to motivate Eq. (29). The procedure is somewhat delicate and we only outline the main steps. Firstly, we expand this equation in powers of the Froude number followed by an expansion in powers of $1 / \sqrt{r}$ when $r \rightarrow \infty$. We then gather the terms that decay slower with $r$-those proportional to $1 / \sqrt{r}$ (the other terms are discarded, that is, we make the far field approximation). Secondly, we expand the resulting function (the amplitude of the spatial dependent trigonometric function) around $\theta^{\prime}=\pi$ and obtain a function $f\left(\theta^{\prime}\right)$. Since we seek the maximum of the potential, we take the derivative of $f\left(\theta^{\prime}\right)$ and identify its zero $f^{\prime}\left(\theta^{\prime}\right)=0$. Solving the previous equation for $\theta^{\prime}$ gives the position of the first maximum of the potential, which within the approximation of Eq. (39), reads $\theta^{\prime}=\pi \pm \sqrt{5} /\left(2 \mathrm{Fr}_{\mathrm{pl}}\right)$. This has the 


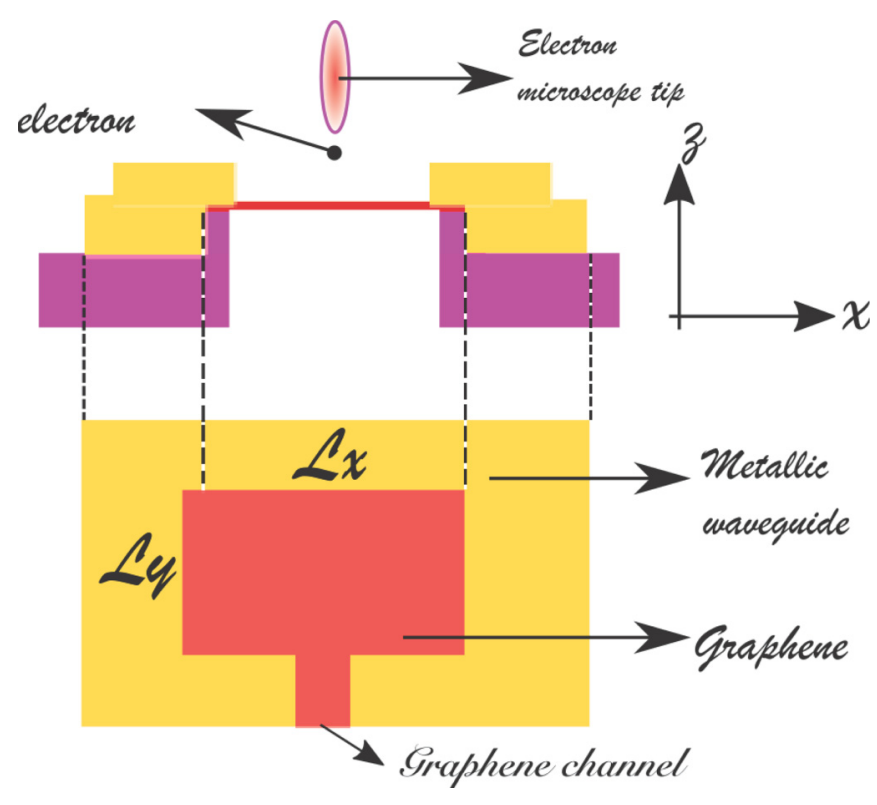

FIG. 9. A graphene sheet enclosed in a metallic waveguide and suspended over a trench. The tip of an electronic microscope injects electrons in the waveguide and the passing through electron excites localized plasmons in graphene. If one bores a hole-graphene channel-in the metallic waveguide and let graphene occupy the hole and connects it to an external graphene sheet, this architecture can act as a source of graphene plasmons.

correct order of magnitude we found from the fit made in Fig. 6.

\section{SOURCE OF GRAPHENE PLASMONS}

Next, we return to the problem of a charged particle moving perpendicularly to a graphene sheet. In this section we consider a rectangular waveguide, of cross-section area $A=L_{x} L_{y}$, where $L_{x}$ and $L_{y}$ are the sides of the rectangle, as depicted in Fig. 9. A microrectangle of graphene is in the middle of the waveguide and an electron is sent along the axis of the waveguide. The waveguide will support discrete graphene plasmonic modes, which can be excited by the passing electron. The goal of this section is to determine the EEL spectrum of the microrectangle of graphene. This method will allow to probe and excite discrete plasmonic resonances in graphene.

This architecture can also be used as a source of plasmons. The idea is conceptually simple: a hole is bored in the metallic waveguide, such that a graphene ribbon extends itself outside the waveguide and connects to an external graphene sheet. The plasmons, once excited in the suspended graphene drum skin will propagate away from the drum though the channel connecting the drum to the external graphene sheet. Choosing drums of different shapes and sizes allows to span a vast spectral range of graphene plasmons. Note that the graphene drum supports in-plane oscillations (compressible charge-density waves), rather than out-of-plane displacements (of the skin) common to the classical sound drum.
For solving this problem, we have to consider, in addition to the hydrodynamic model, equations (B9), the boundary conditions introduced by the waveguide walls, that is,

$$
\begin{gathered}
\left.\frac{\partial \Phi}{\partial x}\right|_{y=0}=\left.\frac{\partial \Phi}{\partial x}\right|_{y=L_{y}}=0, \\
\left.\frac{\partial \Phi}{\partial y}\right|_{x=0}=\left.\frac{\partial \Phi}{\partial y}\right|_{x=L_{x}}=0, \\
v_{y}(y=0)=v_{y}\left(y=L_{y}\right)=v_{x}(x=0)=v_{x}\left(x=L_{x}\right)=0 .
\end{gathered}
$$

In other words, we consider a perfect metallic conductor so that the tangential component of the electric field is null at the waveguide walls and the perpendicular component of the electronic current is null at the graphene boundary.

\section{A. Discrete plasmon dispersion}

Firstly, we consider the plasmonic solutions of the hydrodynamic model. The potential can be conveniently expanded in a Fourier series as

$$
\Phi_{\text {in }}(x, y, z)=\sum_{n, m=1}^{\infty} A_{n m} \sin \left(\frac{n \pi x}{L_{x}}\right) \sin \left(\frac{m \pi y}{L_{y}}\right) e^{-k_{n m}|z|},
$$

where

$$
k_{n m}=\sqrt{\left(\frac{n \pi}{L_{x}}\right)^{2}+\left(\frac{m \pi}{L_{y}}\right)^{2}} .
$$

Integrating Poisson's equation (B9b) with respect to the $z$ coordinate, we have

$$
\begin{aligned}
n_{1}(x, y, \omega) & =-\frac{2 \varepsilon_{0}}{e} \sum_{n, m=1}^{\infty} A_{n m}(\omega) k_{n m} \\
& \times \sin \left(\frac{n \pi x}{L_{x}}\right) \sin \left(\frac{m \pi y}{L_{y}}\right),
\end{aligned}
$$

and the velocity components can be calculated with Eqs. (B9c) and $(44 c)$ :

$$
\begin{aligned}
& v_{x}(x, y)=\frac{2 \varepsilon_{0}}{e} \frac{i \omega}{n_{0}} \sum_{n, m=1}^{\infty} B_{n m} \cos \left(\frac{n \pi x}{L_{x}}\right) \sin \left(\frac{m \pi y}{L_{y}}\right), \\
& v_{y}(x, y)=\frac{2 \varepsilon_{0}}{e} \frac{i \omega}{n_{0}} \sum_{n, m=1}^{\infty} C_{n m} \sin \left(\frac{n \pi x}{L_{x}}\right) \cos \left(\frac{m \pi y}{L_{y}}\right),
\end{aligned}
$$

with the amplitudes $A_{n m}, B_{n m}$, and $C_{n m}$ related via

$$
A_{n m} k_{n m}=B_{n m} \frac{n \pi}{L_{x}}+C_{n m} \frac{m \pi}{L_{y}} .
$$

Lastly, using Eq. (B9), the discretized version of the plasmon dispersion relation (B15) follows:

$$
2 \alpha E_{F} \hbar c k_{n m}+\frac{v_{F}^{2} \hbar^{2}}{2} k_{n m}^{2}=\hbar^{2} \omega_{n m}^{2} .
$$


Quite intuitively, this solution for the plasmons spectrum describes localized surface plasmons in the graphene sheet, in the form of standing waves.

\section{B. Motion of a charge along the axis of symmetry of the waveguide: Charge density fluctuation and EEL spectrum}

Following the steps of Sec. III A, we consider the presence of an electron, moving parallel to the axis of the waveguide, and impinging perpendicularly on the graphene sheet. The density of external charge is given by

$$
\rho_{\mathrm{ext}}=Z e \delta\left(x-x_{0}\right) \delta\left(y-y_{0}\right) \delta(z-v t),
$$

which corresponds to a charged particle with velocity $v$ aimed at the point $\left(x_{0}, y_{0}\right)$ on the graphene surface.

The electric potential follows from solving Poisson's equation $\nabla^{2} \phi_{\text {ext }}=-\rho_{\text {ext }} / \varepsilon_{0}$ with the boundary condition (44):

$$
\begin{aligned}
\Phi_{\mathrm{ext}}(x, y, z, \omega) & =\frac{4 Z e}{\varepsilon_{0} L_{x} L_{y} v} \sum_{n, m=1}^{\infty} \phi_{n m}(z) \sin \left(\frac{n \pi x}{L_{x}}\right) \\
& \times \sin \left(\frac{m \pi y}{L_{y}}\right),
\end{aligned}
$$

with

$$
\left(\partial_{z}^{2}-k_{n m}^{2}\right) \phi_{n m}(z)=-u_{n m} e^{-i \omega z / v} .
$$

Here we have used the completeness relation to rewrite the Dirac delta function as

$$
\delta\left(x-x_{0}\right)=\frac{2}{L_{x}} \sum_{n=1}^{\infty} \sin \left(\frac{n \pi x}{L_{x}}\right) \sin \left(\frac{n \pi x_{0}}{L_{x}}\right),
$$

with a similar expression for $\delta\left(y-y_{0}\right)$, and we have defined $u_{n m}=\sin \left(n \pi x_{0} / L_{x}\right) \sin \left(m \pi y_{0} / L_{y}\right)$.

Equation (53) can be solved with the Green's function method as in Eq. (3), leading to

$$
\phi_{n m}(z)=-\frac{u_{n m}}{(\omega / v)^{2}+k_{n m}^{2}} e^{-i \omega z / v} .
$$

Taking the divergence of Eq. (1a), with the density given by the Fourier expansion (47) and using the continuity equation after a Fourier transform in time, we arrive at

$$
\begin{gathered}
\frac{2 \varepsilon_{0}}{e} \sum_{n, m=1} A_{n m}\left(\omega^{2}-\omega_{n m}^{2}\right) k_{n m} \sin \left(\frac{n \pi x}{L_{x}}\right) \sin \left(\frac{n \pi y}{L_{y}}\right) \\
=-\frac{n_{0} e v_{F}^{2}}{E_{F}} \nabla^{2} \Phi_{\text {ext }}(z=0),
\end{gathered}
$$

with $\omega_{n m}$ given by Eq. (50). Finally, after projecting Eq. (56) onto one of the basis functions, we find

$$
A_{n m}=8 \Phi_{0} \frac{v}{L_{x} L_{y}} \frac{v_{F}^{2}}{E_{F}} \frac{k_{n m}}{\omega^{2}-\omega_{n m}^{2}} \phi_{n m}(0) .
$$

The knowledge of $A_{n m}$ allows us to first compute $n_{1}(x, y, \omega)$ and from this the determination of $n_{1}(x, y, t)$ is possible after a Fourier transform. The determination of the velocity field requires the knowledge of the coefficients $B_{n m}$ and $C_{n m}$, which can be calculated using the density (47), the velocity (48), the electrostatic potential (45) and (52), into Eq. (B10) and using the dispersion relation (50), giving

$$
\begin{aligned}
B_{n m} & =\frac{n \pi}{L_{x}} \frac{A_{n m}}{k_{n m}}, \\
C_{n m} & =\frac{m \pi}{L_{y}} \frac{A_{n m}}{k_{n m}} .
\end{aligned}
$$

Note that Eq. (B10) is vectorial, thus allowing for the determination of the two previous coefficients.

In Fig. 10, we depict the electronic density $n_{1}(x, y, t)$ on the drum, for ascending times, induced by an electron transversing graphene at a speed of $v=0.1 c$ corresponding to a kinetic energy of $2.5 \mathrm{keV}$. We emphasize that this energy value is of the order of the energy used in SEM imaging of graphene and therefore would cause little to none damage to the material. We stress that the knock-on-threshold for electrons in graphene is much larger, close to $80 \mathrm{keV}$ ). Note in this figure the evolution of the charge density from initially circular concentric waves at the center of the drum (for shorter times) to interference fringes (at larger times), due to reflection at the boundaries of the drum. In the presence of the channel (see Fig. 9), the plasmonic wave reaches the boundary of the drum and some of the plasmon frequency components will propagate through the channel outwards. Note that in Fig. 10, the electron hits the drum at its center. By choosing a different impact point we may relax reflection symmetries of the problem, thus in turn producing more directional waves (results not shown). In passing, we note that such directional waves could also be explored in nonintegrable geometries, such as chaotically shaped billiards, or by turning from graphene to anisotropic $2 \mathrm{D}$ materials.

The EEL spectrum can be calculated using the same definition and method used in the previous section and reads

$$
\Gamma(\omega)=\frac{\Psi_{0}}{\omega} \sum_{n, m=1}^{\infty} \delta\left(\omega-\omega_{n m}\right) \frac{k_{n m}^{2} u_{n m}^{2}}{\left[\omega^{2}+\left(k_{n m} v\right)^{2}\right]^{2}},
$$

with

$$
\Psi_{0}=32 \pi^{2} \alpha^{2} \hbar c^{2} \frac{n_{0} v_{F}^{2} v^{2}}{L_{x} L_{y} E_{F}} .
$$

The emergence of Dirac delta functions in the EEL spectrum guaranties that a single electron can excite multiple plasmon modes, albeit with different weights.

In the limit $L_{x}=L_{y} \rightarrow \infty$, the sums are conveniently converted into an integral:

$\Gamma(\omega)=\frac{\Psi_{0}}{\omega} \frac{L_{x} L_{y}}{\pi^{2}} \int_{0}^{\pi / 2} d \theta \int_{0}^{\infty} k d k \delta\left(\omega-\omega_{k}\right) \frac{k^{2} u^{2}(k, \theta)}{\left[\omega^{2}+(k v)^{2}\right]^{2}}$.

We have to perform the integral over the delta function, which is elementary, and the angular integral, leading to

$$
\begin{aligned}
\Gamma(\omega) & =\Psi_{0} \frac{L_{x} L_{y}}{\pi^{2}} \int_{0}^{\pi / 2} d \theta \frac{2 \omega^{2} u^{2}\left(\omega^{2} / a, \theta\right)}{\left(a^{2}+v^{2} \omega^{2}\right)^{2}} \\
& =\frac{2 \hbar}{E_{F}} \frac{v^{2} \omega^{2} / a^{2}}{\left(1+v^{2} \omega^{2} / a^{2}\right)^{2}} .
\end{aligned}
$$



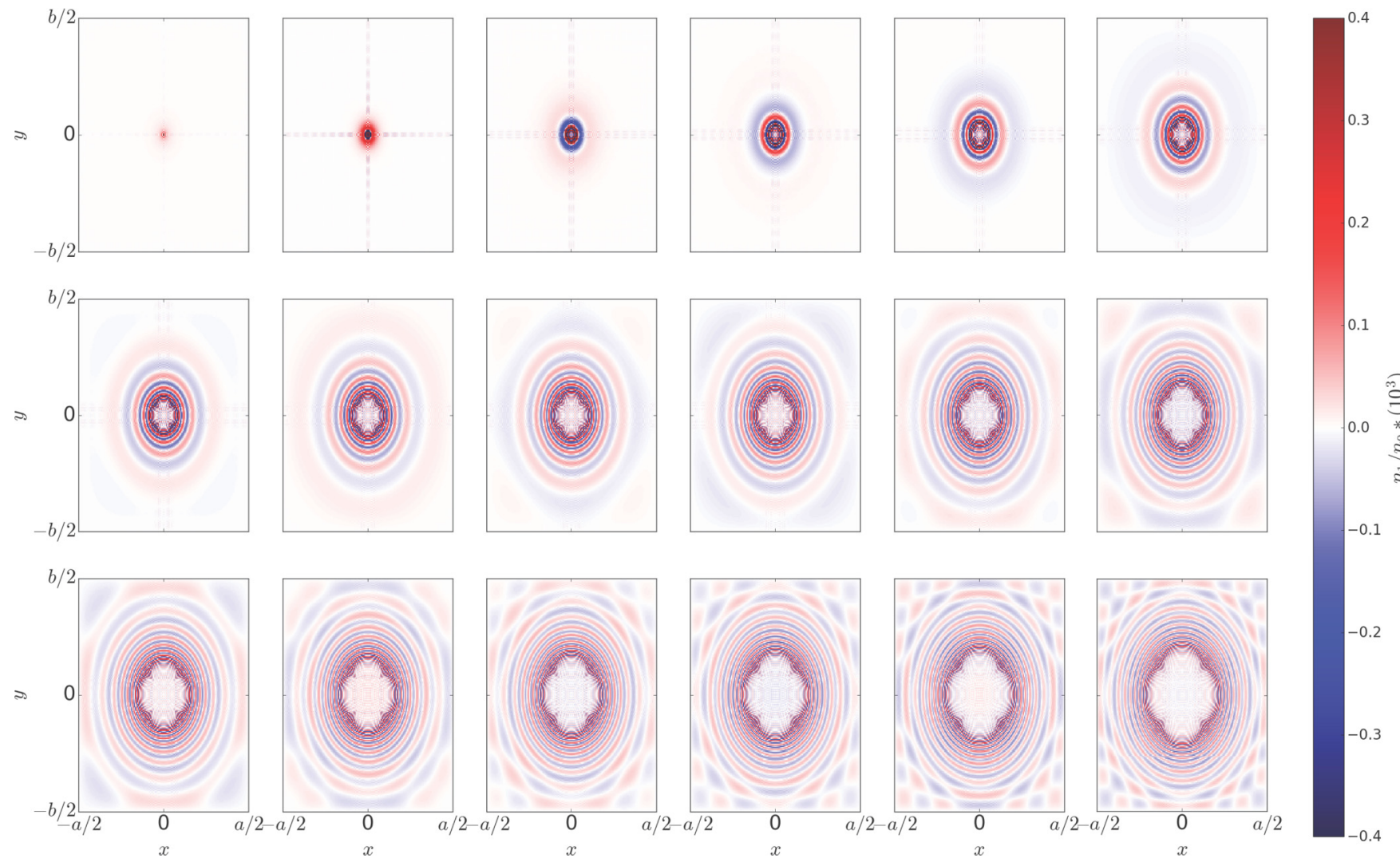

FIG. 10. Induced electronic density fluctuations $n_{1}(x, y)$ in the graphene drum for $t=0.1 \mathrm{fs}+18(n-1)$ fs, where $n$ is the number of the panel (ordered by rows from left to right). The parameters are $v=0.1 c, E_{F}=0.37 \mathrm{eV}$, and $L_{x}=L_{y}=3 \mu \mathrm{m}$. Note the evolution of the density from a circle for short times and the development of interference fringes for larger times. The blue and red colors correspond, respectively, to negative and positive charge density fluctuations $n_{1}(x, y)$ relatively to the homogeneous charge density $n_{0}$. Note that the wavelength of the plasmons when they are first created is much smaller than the size of the drum. It is therefore conceivable that they can escape through a graphene channel drilled in the walls of the waveguide. The figures present a high degree of symmetry because the impact point chosen for the electron is the center of the square.

As before, we have written $\omega_{k}=\sqrt{a k}$ (recall that $a$ has unit of acceleration) and used the limit (choosing $x_{0}=y_{0}=L_{x} / 2$ ),

$$
\lim _{L_{x} \rightarrow \infty} \frac{16}{\pi} \int_{0}^{\pi / 2} d \theta u^{2}\left(\omega^{2} / a, \theta\right)=2 .
$$

We have therefore recovered the result for a continuous graphene sheet, given by Eq. (20).

In Fig. 11, we depict the EEL spectrum of a graphene drum, given by Eq. (59). It is clear from this figure that a single electron can excite more than one surface plasmon mode. This is evident from the presence of several peaks in the EEL spectrum at different frequencies. This effect has already been seen in the excitation of localized plasmons in graphene nanostructures [34]. Also note that there is an optimal speed for the more efficient excitation of plasmons. For the first peak (at low frequencies), the optimal speed is in the interval $v \in[0.1 c, 0.2 c]$.

\section{DISCUSSION AND CONCLUSIONS}

We have considered the problem of the excitation of surface plasmons in graphene by a fast moving charge. We have analyzed two cases: (i) when the charge is moving

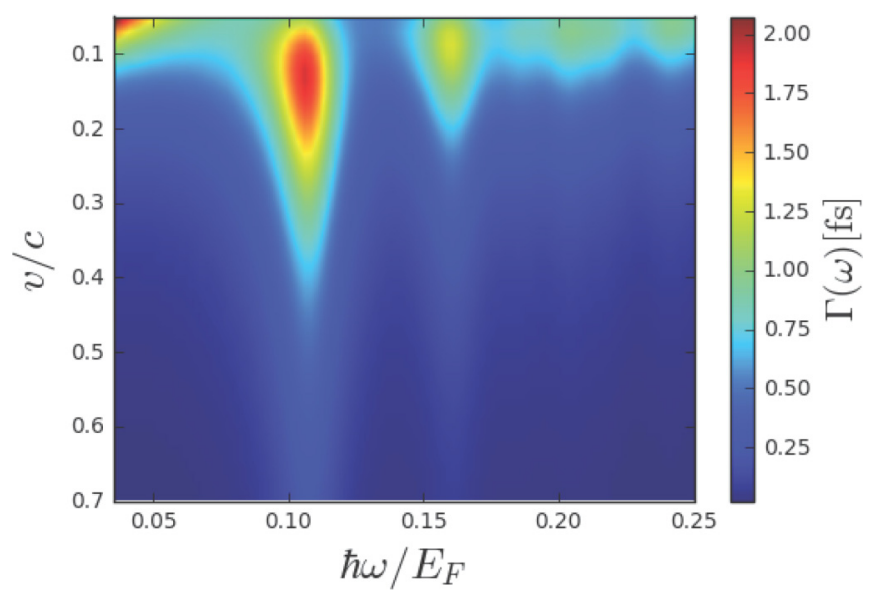

FIG. 11. EEL spectrum of a graphene square drum $\left(L_{x}=L_{y}=\right.$ $3 \mu \mathrm{m})$ as function of the speed of the moving charge and of the frequency. If we draw a horizontal line throughout the figure we obtain the EEL spectrum for a given speed. It is clear that this cut produces a new figure with peaks at certain frequencies, corresponding to the excitation of the localized plasmons in the drum. We have considered a Fermi energy of $E_{F}=0.37 \mathrm{eV}$ and have broaden the delta functions to Lorentzians with a relaxation rate of $4.1 \mathrm{meV}$. 
perpendicular to the graphene sheet and (ii) when the charge is moving parallel to the graphene sheet. In the first case we have computed the EEL spectrum [33] and found that surface plasmons from a continuum of wave numbers are excited by this method. The excitation of surface plasmons of different frequencies has been discussed in the literature before [34] in the context of graphene-based nanostructures. In this reference it is shown that, for continuous graphene, the EEL spectrum has a maximum at (in agreement with our result) $\hbar \omega / E_{F}=2 \alpha c / v$ implying that the frequency maximum shifts to higher frequencies as $v$ decreases, as seen in Fig. 3. Taking, for example, $v / c=0.01$, the previous result implies that $\hbar \omega / E_{F} \approx 1.5$, which agrees with Fig. 3 . As we have seen, in the case of an infinite graphene sheet the EEL spectrum has broad resonances, which is an indication of the simultaneous excitation of plasmons of different energies. In the second case, the moving charge induces a plasmonic wake as it moves over graphene.

Contrary to what could have been expected, the wake induced by the moving charge is not necessarily of Kelvin type and can also be of Mach type, where the angle of the cone of the charge wake is proportional to the inverse of the speed of the moving charge and therefore can be much smaller than the value predicted by Kelvin theory. We have shown that there is a transition Froude number from Kelvin to Mach type of ship wakes. These two different possibilities have been observed in real ship wakes and the transition is controlled by the Froude number [36], Fr $=\sqrt{U^{2} /(L g)}$, where $U$ is the ship speed, $L$ is the hull ship length, and $g$ is the acceleration of gravity. We have noted in our graphene problem, the dimensionless number $a z_{0} / v^{2}$ determines the transition from the Mach-like to Kelvin-like regimes. Therefore we interpret $\mathrm{Fr}_{\mathrm{pl}}=\sqrt{v^{2} /\left(z_{0} a\right)}$ as the Froude number for the plasmonic wakes in graphene, where the "acceleration" $a$ depends on the Fermi energy of graphene, and controls the dispersion of graphene plasmons. The value $\mathrm{Fr}_{\mathrm{pl}} \sim 2$ defines the transition region from Mach-type, $\mathrm{Fr}_{\mathrm{pl}} \gg 1$, to Kelvin-type, $\mathrm{Fr}_{\mathrm{pl}} \ll 1$, regimes.

As noted, we have gathered numerical evidence of a critical Froude number that signals the transition from Mach-type to Kelvin-type of wakes, whose value reads approximately $\mathrm{Fr}_{\mathrm{pl}}^{\mathrm{c}} \approx 2$. This value is about 4 times larger than the critical Froude number in ship wakes [36]. On the other hand, for pointlike objects immersed in a fluid, the critical Froude number has been found to be larger than 2 [39], in agreement with our results. We note that the way the apparent opening angle of the wake is measured does not influence the value of the Froude number at which the transition occurs. See Ref. [39] for a different method from ours of measuring the angle. Interestingly, the parameter $a$ also depends on the dielectric constant of the environment. This introduces an additional degree of freedom, besides the tunning of the Fermi energy, to gain control over the Froude number, a situation that has no parallel in ship wakes.

We end this part of the discussion noting that the group velocity of the plasmons in graphene reads $v_{g}=d \sqrt{a k} / d k=$ $\frac{1}{2} \sqrt{a / k}=\frac{1}{2} \sqrt{a \lambda_{\mathrm{sp}} /(2 \pi)}$. On the other hand, gravity waves in deep waters propagate with a group velocity $v_{w}=$ $\frac{1}{2} \sqrt{g \lambda /(2 \pi)}$. We note that $v_{g}$ and $v_{w}$ are identical, except that in $v_{g}$ we have $\lambda_{\text {sp }}$ being the wavelength of the surface plasmon. It is therefore not so surprising that the same physics we find in ship wakes has also been found in surface plasmon wakes as long as the distance of the charged particle to the graphene sheet $z_{0}$ plays the same role as the hull ship length $L$. This latter possibility was not evident from the outset.

Our calculations are valid for suspended graphene. It would be interesting to extend them for graphene on hexagonal boron nitride for discussing the excitation of phonon-plasmonpolaritons. This is an important topic for nanophotonics [40]. Also, extending this work to multilayer graphene [41] is a natural continuation of this work.

We have also discussed the formation of localized plasmons in a graphene drum in a metallic waveguide. As noted, the system can be used as a plasmon source of collimated beam. We explored the rectangular drum, but a circular drum is also feasible and it will originate plasmons of different frequencies. We note that since for waveguides of the order of $1 \mu \mathrm{m}$ its cutoff frequency is in the near-IR, the electron, once in the waveguide, can only radiate above this cutoff frequency. Therefore all radiation that appears below this frequency is plasmonic in nature and there will be no transition radiation in that frequency range, at least for deep enough waveguides, compared with the plasmon confining length in the transverse direction. Also, we have considered the simple case where the electron impinges at the center of the square. If we had considered a different impinging point then we would have created highly directional plasmons oriented toward the channel that conducts the plasmons out of the drum. If below graphene we deposit a metallic film at a distance of one or two layers of hexagonal boron nitride, then graphene will support strongly confined plasmons, akin to acoustic plasmons in a continuous graphene sheet. This setup would work as a source of acoustic plasmons.

We can consider the graphene drum a plasmonic billiard, which presents a characteristic spectrum distribution as function of frequency. Both in classical and quantum billiards, it is well known that depending on their geometry, the trajectories (classical) and spectrum (quantum) can be chaotic. It would be interesting to study the spectrum distribution of plasmonic billiards in the future.

Note added. After the submission of this paper we became aware of a similar work [42], but in the field of fluid dynamics.

\section{ACKNOWLEDGMENTS}

A.J.C. acknowledges for a scholarship from the Brazilian agency $\mathrm{CNPq}$ (Conselho Nacional de Desenvolvimento Científico e Tecnológico). N.M.R.P. acknowledges useful discussions with Jaime Santos and support from the European Commission through the project "Graphene-Driven Revolutions in ICT and Beyond" (Ref. No. 696656) and the Portuguese Foundation for Science and Technology (FCT) in the framework of the Strategic Financing UID/FIS/04650/2013. The Center for Nanostructured Graphene (CNG) was financed by the Danish National Research Council (DNRF103). N.A.M. is a VILLUM Investigator supported by VILLUM Fonden (Grant No. 16498). 


\section{APPENDIX A: FROM BOLTZMANN EQUATION TO EULER'S EQUATION OF HYDRODYNAMICS}

In this section, we derive Euler's equation for fluid motion starting from the Boltzmann equation. Let us assume an electron gas characterized by the distribution function $f(\mathbf{r}, \mathbf{v}) d \mathbf{r} d \mathbf{v}$, which specifies the number of particles in the gas having position and velocity centered at $\mathbf{r}$ and $\mathbf{v}$, respectively, in the small volume $d \mathbf{r}$ and in the small velocity range $d \mathbf{v}$. We can introduce a six-dimensional phase-space vector $\mathbf{w}=(\mathbf{r}, \mathbf{v})$ whose time rate reads $\dot{\mathbf{w}}=(\dot{\mathbf{r}}, \dot{\mathbf{v}})$. If the forces are conservative then $\dot{\mathbf{v}}=-\nabla \Phi$, where $\Phi$ is the potential energy per unit mass. The time evolution of the distribution function $f(\mathbf{r}, \mathbf{v})$ is given by the Boltzmann equation [43]

$$
\frac{\partial f}{\partial t}+\mathbf{v} \cdot \frac{\partial f}{\partial \mathbf{r}}+\mathbf{g} \cdot \frac{\partial f}{\partial \mathbf{v}}=0
$$

where collisions have been excluded, and $\mathbf{g}$ represents the external forces per unit mass. The previous equation is called the collisionless Boltzmann equation. Adding collisions amounts to adding a term of the form $\gamma(t)$ to the right-hand side of this equation. Boltzmann's equation is six-dimensional in phase space and has more information that we actually need. Since we want to know the position of the particles as function of time we can integrate Boltzmann equation over the coordinate v. Next, we will compute the first and second moments of the Boltzmann equation. To that end, we introduce the mass density using the relation [43] (we are assuming all particles equal with mass $m$ )

$$
\rho(\mathbf{r})=\int d \mathbf{v} f(\mathbf{r}, \mathbf{v}) m
$$

and the velocity moment via [43]

$$
\left\langle v_{i}\right\rangle=\frac{1}{\rho(\mathbf{r})} \int d \mathbf{v} f(\mathbf{r}, \mathbf{v}) m v_{i} .
$$

It is also convenient to introduce the second moment of the velocity as [43]

$$
\left\langle v_{i} v_{j}\right\rangle=\frac{1}{\rho(\mathbf{r})} \int d \mathbf{v} f(\mathbf{r}, \mathbf{v}) m v_{i} v_{j} .
$$

Let us now take the zero moment of the Boltzmann equation

$$
\int d \mathbf{v}\left[\frac{\partial f(\mathbf{r}, \mathbf{v})}{\partial t}+\mathbf{v} \cdot \frac{\partial f(\mathbf{r}, \mathbf{v})}{\partial \mathbf{r}}+\mathbf{g} \cdot \frac{\partial f(\mathbf{r}, \mathbf{v})}{\partial \mathbf{v}}\right]=0
$$

from where it follows the continuity equation

$$
\frac{\partial \rho(\mathbf{r})}{\partial t}+\frac{\partial}{\partial \mathbf{r}} \cdot[\rho(\mathbf{r})\langle\mathbf{v}\rangle]=0
$$

stating mass conservation, and where we have used the divergence theorem leading to the following identity:

$$
\int_{V} d \mathbf{v} \frac{\partial f(\mathbf{r}, \mathbf{v})}{\partial \mathbf{v}}=\int_{S_{\infty}} f(\mathbf{r}, \mathbf{v}) d \mathbf{S}_{\mathbf{v}}=0,
$$

where $f(\mathbf{r}, \mathbf{v})=0$ over a surface at infinity, $S_{\infty}$.

Let us next consider the first moment of the Boltzmann equation

$$
\int d \mathbf{v} \mathbf{v}\left[\frac{\partial f(\mathbf{r}, \mathbf{v})}{\partial t}+\mathbf{v} \cdot \frac{\partial f(\mathbf{r}, \mathbf{v})}{\partial \mathbf{r}}+\mathbf{g} \cdot \frac{\partial f(\mathbf{r}, \mathbf{v})}{\partial \mathbf{v}}\right]=0,
$$

which can be simplified to

$$
\frac{\partial}{\partial t}[\rho(\mathbf{r})\langle\mathbf{v}\rangle]+\sum_{i} \frac{\partial}{\partial x_{i}}\left[\rho(\mathbf{r})\left\langle\mathbf{v} v_{i}\right\rangle\right]-\mathbf{g} \rho(\mathbf{r})=0,
$$

where the third term was computed using integration by parts. This last equation is called the momentum equation. We now introduce the tensor $\tau_{i j}^{2}=\left\langle v_{i} v_{j}\right\rangle-\left\langle v_{i}\right\rangle\left\langle v_{j}\right\rangle$. This conveniently allows us to write the term with the second moment in terms of products of first moments. Subtracting from the momentum equation the continuity equation, we obtain for each component

$$
\rho(\mathbf{r}) \frac{\partial\left\langle v_{j}\right\rangle}{\partial t}+\rho(\mathbf{r}) \sum_{i}\left\langle v_{i}\right\rangle \frac{\partial\left\langle v_{j}\right\rangle}{\partial x_{i}}=g_{j} \rho(\mathbf{r})-\sum_{i} \frac{\partial\left[\rho(\mathbf{r}) \tau_{i j}^{2}\right]}{\partial x_{i}}
$$

or in vectorial terms

$$
\rho(\mathbf{r}) \frac{\partial\langle\mathbf{v}\rangle}{\partial t}+\rho(\mathbf{r})(\langle\mathbf{v}\rangle \cdot \nabla)\langle\mathbf{v}\rangle=\mathbf{g} \rho(\mathbf{r})-\nabla P,
$$

where $P$ is the pressure in the gas. The last equation is Euler's equation of fluids dynamics and is the starting point for the hydrodynamic model of plasmons in metals and in graphene. We should stress that the derivation of Eq. (A11) assumed a finite effective mass $m$ for the particles in the gas. Adapting this equation for graphene will require the introduction of graphene electrons' Drude mass $m_{g}=\hbar k_{F} / v_{F}$, which links the Fermi momentum $\hbar k_{F}$ to the Fermi velocity $v_{F}$; both well-defined properties associated with the linear dispersion of massless Dirac fermions in graphene. This choice makes sense since we are describing transport properties: for a 2D electron gas with quadratic dispersion, the Drude conductivity depends on the effective mass $m$ of the electron, whereas in graphene the same quantity depends on the mass $m_{g}$. Therefore it is permissible to replace $m$ by $m_{g}$ in the hydrodynamic equation [21]. A note is in order here: we have formulated the problem with the aid of velocity fields which is a natural choice for massive particles. Naturally, we could also have formulated the equation of motion in terms of the momentum fields in which case we would have arrived at the same final equation, but without the need to assign a Drude mass to the electrons in the graphene.

\section{APPENDIX B: HYDRODYNAMIC MODEL FOR DOPED GRAPHENE IN THE ELECTROSTATIC REGIME}

The hydrodynamic model of the electromagnetic response of an electron gas couples Euler's equation (see Appendix A for a derivation starting with Boltzmann's equation) to Maxwell's equations [20,21,26,27]. To emphasize the $2 \mathrm{D}$ nature of the problem, we will in the following introduce $\mathbf{r}=\left(\mathbf{r}_{\|}, z\right)$ with $\mathbf{r}_{\|}$ being a $2 \mathrm{D}$ position vector in the plane of the graphene $(z=0)$, while $z$ is in the direction perpendicular to the graphene layer. If we introduce the density of particles in the gas per unit area as $n\left(\mathbf{r}_{\|}\right)$then the mass density is defined as $\rho\left(\mathbf{r}_{\|}\right)=m n\left(\mathbf{r}_{\|}\right)$, where $m$ is the mass of the particle and $\mathbf{r}_{\|}$is the 2D position vector. In terms of $n\left(\mathbf{r}_{\|}\right)$Euler's equation reads (where we have dropped the average symbol for simplicity; see Appendix A)

$$
m n\left(\mathbf{r}_{\|}\right) \frac{\partial \mathbf{v}}{\partial t}+m n\left(\mathbf{r}_{\|}\right)(\mathbf{v} \cdot \nabla) \mathbf{v}=\mathbf{g} m n\left(\mathbf{r}_{\|}\right)-\nabla P .
$$


If scattering is allowed, then a term of the form $m n(\mathbf{r}) \mathbf{v} / \tau$ must be included in the left-hand side of the previous equation, and where $\tau$ is a relaxation time taking into account the nonconservation of momentum. The physics of this added term stems for the electron-phonon and electron-electron interactions. We now consider that there is an external electric field applied to the electron gas. This changes Euler's equation to

$$
m n\left(\mathbf{r}_{\|}\right) \frac{\partial \mathbf{v}}{\partial t}+m n\left(\mathbf{r}_{\|}\right)(\mathbf{v} \cdot \nabla) \mathbf{v}=e n\left(\mathbf{r}_{\|}\right) \nabla \phi\left(\mathbf{r}_{\|}, z=0\right)-\nabla P,
$$

where $e$ is the elementary charge, $\phi(\mathbf{r})=\phi\left(\mathbf{r}_{\|}, z\right)$ is the electrostatic potential, and the electric field follows from $\mathbf{E}=-\nabla \phi(\mathbf{r})$.

Since we are dealing with an electron gas, the simplest approximation for the pressure is the statistical pressure encoded in the kinetic energy of the electron gas. For graphene, the determination of the pressure (force per unit length in 2D) requires the calculation of the kinetic energy of the noninteracting gas as

$$
K_{g}=4 A \int \frac{d \theta k d k}{(2 \pi)^{2}} v_{F} \hbar k=v_{F} \hbar \frac{2}{3 \pi} \pi^{3 / 2} N_{e}^{3 / 2} A^{-1 / 2},
$$

where $N_{e}$ in the total number of electrons, $A$ is the area of the system, and we have expressed the Fermi momentum in graphene by $k_{F}=\sqrt{\pi n_{0}}$, where $n_{0}$ is the 2D particle density. It follows that the pressure is given by

$$
P=-\frac{\partial K_{g}}{\partial A}=v_{F} \hbar \frac{1}{3 \pi}\left(\pi n_{0}\right)^{3 / 2} .
$$

Note that the previous quantity is a $2 \mathrm{D}$ pressure. Next, we consider that the pressure in the inhomogeneous gas has the same functional form in terms of density as that given by the previous equation. Using this assumption, we can now compute the gradient of the pressure as

$$
\nabla P=v_{F} \hbar \frac{1}{2} \sqrt{\pi n\left(\mathbf{r}_{\|}\right)} \nabla n\left(\mathbf{r}_{\|}\right) .
$$

Dividing the pressure by the graphene's Drude mass we obtain Euler's equation of motion for graphene as

$$
\frac{\partial \mathbf{v}}{\partial t}+(\mathbf{v} \cdot \nabla) \mathbf{v}=\frac{e v_{F}}{\hbar k_{F}} \nabla \phi\left(\mathbf{r}_{\|}, 0\right)-\frac{v_{F}^{2}}{2 n\left(\mathbf{r}_{\|}\right)} \nabla n\left(\mathbf{r}_{\|}\right),
$$

where the electrostatic potential depends on $\mathbf{r}$ but is evaluated at the point in graphene given by $\mathbf{r}=\left(\mathbf{r}_{\|}, 0\right)$. The other two equations defining the hydrodynamic model in the electrostatic limit are Poisson's equation

$$
\nabla^{2} \phi=-\frac{e}{\epsilon_{0}}\left[n_{+}-n\left(\mathbf{r}_{\|}\right)\right] \delta(z),
$$

where $n_{+}$is the ionic charge density neutralizing the electron gas, and the continuity equation

$$
\frac{\partial n\left(\mathbf{r}_{\|}\right)}{\partial t}+\nabla \cdot\left[n\left(\mathbf{r}_{\|}\right) \mathbf{v}\right]=0
$$

stating charge conservation in the graphene sheet.

We now linearize the hydrodynamic equations, assuming $n\left(\mathbf{r}_{\|}\right) \approx n_{0}+n_{1}\left(\mathbf{r}_{\|}\right)$(we note in passing that for metals we have $n_{0} \gg n_{1}$; for graphene, however, this is not the case when the system is near the neutrality point; in this paper, we will be far from this regime) and $\phi\left(\mathbf{r}_{\|}\right) \approx \phi_{0}\left(\mathbf{r}_{\|}\right)+\phi_{1}\left(\mathbf{r}_{\|}\right)$, and noting that $\mathbf{v}$ is already a linear order quantity. This leads to the linear hydrodynamic model

$$
\begin{aligned}
\frac{\partial \mathbf{v}}{\partial t} & =\frac{e v_{F}}{\hbar k_{F}} \nabla \phi_{1}\left(\mathbf{r}_{\|}, 0\right)-\frac{v_{F}^{2}}{2 n_{0}} \nabla n_{1}\left(\mathbf{r}_{\|}\right), \\
\nabla^{2} \phi_{1}(\mathbf{r}) & =\frac{e}{\epsilon_{0}} \delta(z) n_{1}\left(\mathbf{r}_{\|}, 0\right), \\
0 & =\frac{\partial n_{1}\left(\mathbf{r}_{\|}\right)}{\partial t}+n_{0} \nabla \cdot \mathbf{v} .
\end{aligned}
$$

Note that the second term on the right-hand side of Eq. (B9a) is proportional to $1 / n_{0}$ and therefore can rightfully be considered a correction to the first term. Indeed, we can rewrite this equation as

$$
\frac{\partial \mathbf{v}}{\partial t}=\frac{e v_{F}^{2}}{E_{F}} \nabla \phi_{1}\left(\mathbf{r}_{\|}, 0\right)-\frac{\pi}{2} \frac{\hbar^{2} v_{F}^{4}}{E_{F}^{2}} \nabla n_{1}\left(\mathbf{r}_{\|}\right),
$$

which shows that the second term on the right-hand side of this equation is of higher order in powers of $1 / E_{F}$. Also the presence of $\hbar^{2}$ in the second term signals the presence of a correction of quantum nature.

\section{Spectrum of the surface plasmons}

For solving the previous three equations, we introduce the Fourier transform in the plane (note that here $\mathbf{k}$ is the in-plane 2D wave vector),

$$
\mathbf{v}\left(\mathbf{r}_{\|}, t\right)=\int \frac{d \omega d \mathbf{k}}{(2 \pi)^{3}} \mathbf{v}(\mathbf{k}, \omega) e^{i\left(\mathbf{k} \cdot \mathbf{r}_{\|}-\omega t\right)},
$$

and equivalent definitions for the pairs of transforms $\left[n_{1}\left(\mathbf{r}_{\|}, t\right) ; n_{1}(\mathbf{k}, \omega)\right]$ and $\left[\phi_{1}\left(\mathbf{r}_{\|}, z, t\right) ; \phi_{1}(\mathbf{k}, z, \omega)\right]$. Using the Fourier transforms in the hydrodynamic equations, we obtain

$$
-i \omega \mathbf{v}(\mathbf{k}, \omega)=\frac{e v_{F}}{\hbar k_{F}} i \mathbf{k} \phi_{1}(\mathbf{k}, 0, \omega)-\frac{v_{F}^{2}}{2 n_{0}} i \mathbf{k} n_{1}(\mathbf{k}, \omega)
$$

for Euler's equation,

$$
\left(\frac{\partial^{2}}{\partial z^{2}}-k^{2}\right) \phi_{1}(\mathbf{k}, z, \omega)=\frac{e}{\epsilon_{0}} \delta(z) n_{1}(\mathbf{k}, \omega)
$$

for Poisson's equation, and

$$
0=-i \omega n_{1}(\mathbf{k}, \omega)+n_{0} i \mathbf{k} \cdot \mathbf{v}(\mathbf{k}, \omega)
$$

for the continuity equation. Note that Eq. (B12b) is nothing but the Green's function. For obtaining $\phi_{1}(\mathbf{k}, z, \omega)$, we assume that $\phi_{1}(\mathbf{k}, z, \omega)=A e^{-k z}$, for $z>0$ and $\phi_{1}(\mathbf{k}, z, \omega)=B e^{k z}$ for $z<$ 0 . The coefficients $A$ and $B$ are determined from the boundary conditions: $A=B$ and $-k(A+B)=\frac{e}{\epsilon_{0}} n_{1}(\mathbf{k}, \omega)$, which imply that

$$
A=-\frac{e}{2 k \epsilon_{0}} n_{1}(\mathbf{k}, \omega) .
$$

Using the last result in Eq. (B12a), it follows a relation between $\mathbf{v}(\mathbf{k}, \omega)$ and $n_{1}(\mathbf{k}, \omega)$. Using this relation in the continuity equation (B13), we obtain

$$
\hbar^{2} \omega_{\mathrm{spp}}^{2}=\left(2 \alpha E_{F} \hbar c k+\frac{v_{F}^{2} \hbar^{2}}{2} k^{2}\right) \approx 2 \alpha E_{F} \hbar c k,
$$

where $\alpha$ is the fine structure constant, with the approximate result valid for realistic $\left(k<k_{F}\right)$ wave numbers. We have, 
therefore, recovered the well-known result for the square-root dispersion of graphene surface plasmons in the electrostatic limit [44]. This is consistent with a small-wave-number expansion of the intraband part of the RPA result.
[1] A. D. Boardman, Hydrodynamic Theory of Plasmons on Plane Surfaces, Electromagnetic Surface Modes (Wiley, Chichester, 1982).

[2] A. Moreau, C. Ciracì, and D. R. Smith, Impact of nonlocal response on metallodielectric multilayers and optical patch antennas, Phys. Rev. B 87, 045401 (2013).

[3] C. Ciracì, J. B. Pendry, and D. R. Smith, Hydrodynamic model for plasmonics: A macroscopic approach to a microscopic problem, ChemPhysChem 14, 1109 (2013).

[4] G. Toscano, S. Raza, A.-P. Jauho, N. A. Mortensen, and M. Wubs, Modified field enhancement and extinction by plasmonic nanowire dimers due to nonlocal response, Opt. Express 20, 4176 (2012).

[5] A. D. Boardman, B. V. Paranjape, and Y. O. Nakamura, Surface plasmon-polaritons in a spatially dispersive inhomogeneous medium, Physica Status Solidi B 75, 347 (1976).

[6] A. D. Boardman and R. Ruppin, The boundary conditions between spatially dispersive media, Surf. Sci. 112, 153 (1981).

[7] Y. Luo, A. I. Fernandez-Dominguez, A. Wiener, S. A. Maier, and J. B. Pendry, Surface Plasmons and Nonlocality: A Simple Model, Phys. Rev. Lett. 111, 093901 (2013).

[8] T. Christensen, W. Yan, S. Raza, A.-P. Jauho, N. A. Mortensen, and M. Wubs, Nonlocal response of metallic nanospheres probed by light, electrons, and atoms, ACS Nano 8, 1745 (2014).

[9] A. Aldea and V. Bârsan, Trends in Nanophysics: Theory, Experiment and Technology (Springer, Berlin, 2010).

[10] S. Raza, T. Christensen, M. Wubs, S. I. Bozhevolnyi, and N. A. Mortensen, Nonlocal response in thin-film waveguides: Loss versus nonlocality and breaking of complementarity, Phys. Rev. B 88, 115401 (2013).

[11] A. N. Grigorenko, M. Polini, and K. S. Novoselov, Graphene plasmonics, Nat. Photon. 6, 749 (2012).

[12] A. L. Fetter, Electrodynamics of a layered electron gas. I. single layer, Ann. Phys. 81, 367 (1973).

[13] H. Shen, L. Chen, L. Ferrari, M.-H. Lin, N. A. Mortensen, S. Gwo, and Z. Liu, Optical observation of plasmonic nonlocal effects in a 2D superlattice of ultrasmall gold nanoparticles, Nano Lett. 17, 2234 (2017).

[14] W. Wang and J. M. Kinaret, Plasmons in graphene nanoribbons: Interband transitions and nonlocal effects, Phys. Rev. B 87, 195424 (2013).

[15] S. Raza, S. I. Bozhevolnyi, M. Wubs, and N. A. Mortensen, Nonlocal optical response in metallic nanostructures, J. Phys.: Condens. Matter 27, 183204 (2015).

[16] Y. Luo, R. Zhao, and J. B. Pendry, van der Waals interactions at the nanoscale: The effects of nonlocality, Proc. Natl. Acad. Sci. USA 111, 18422 (2014).

[17] C. Ciracı, R. T. Hill, J. J. Mock, Y. Urzhumov, A. I. FernándezDomínguez, S. A. Maier, J. B. Pendry, A. Chilkoti, and D. R. Smith, Probing the ultimate limits of plasmonic enhancement, Science 337, 1072 (2012).

[18] M. Müller, J. Schmalian, and L. Fritz, Graphene: A Nearly Perfect Fluid, Phys. Rev. Lett. 103, 025301 (2009).
[19] M. Mendoza, H. J. Herrmann, and S. Succi, Hydrodynamic model for conductivity in graphene, Sci. Rep. 3, 1052 (2013).

[20] S. Raza, Plasmonic Nanostructures: A hydrodynamic Approach, Master's thesis, Technical University of Denmark, 2011.

[21] T. Christensen, From Classical to Quantum Plasmonics in Three and Two Dimensions (Springer, Berlin, 2017).

[22] D. Huang, G. Gumbs, P. M. Alsing, and D. A. Cardimona, Nonlocal mode mixing and surface-plasmon-polariton-mediated enhancement of diffracted terahertz fields by a conductive grating, Phys. Rev. B 77, 165404 (2008).

[23] C. David and F. Javier García de Abajo, Surface plasmon dependence on the electron density profile at metal surfaces, ACS-Nano 8, 9558 (2014).

[24] M. B. Lundeberg, Y. Gao, R. Asgari, C. Tan, B. Van Duppen, M. Autore, P. Alonso-Gonzalez, A. Woessner, K. Watanabe, T. Taniguchi, R. Hillenbrand, J. Hone, M. Polini, and F. H. L. Koppens, Tuning quantum nonlocal effects in graphene plasmonics, Science 357, 187 (2017).

[25] T. Christensen, W. Wang, A.-P. Jauho, M. Wubs, and N. A. Mortensen, Classical and quantum plasmonics in graphene nanodisks: Role of edge states, Phys. Rev. B 90, 241414(R) (2014).

[26] J. M. Pitarke, V. M. Silkin, E. V. Chulkov, and P. M. Echenique, Theory of surface plasmons and surface-plasmon polaritons, Rep. Prog. Phys. 70, 1 (2007).

[27] M. Scalora, M. A. Vincenti, D. de Ceglia, V. Roppo, M. Centini, N. Akozbek, and M. J. Bloemer, Second- and third-harmonic generation in metal-based structures, Phys. Rev. A 82, 043828 (2010).

[28] V. Fessatidis, N. J. M. Horing, and A. Balassis, Power loss of an energetic charged particle moving parallel to a graphene sheet, Phys. Lett. A 375, 192 (2010).

[29] N. J. M. Horing and H. C. Tso, Fast-particle energy loss in the vicinity of a two-dimensional plasma, Phys. Rev. B 36, 1588 (1987).

[30] G. Gumbs, Fast-particle energy loss to a layered electron gas, Phys. Rev. B 37, 10184 (1988)

[31] G. Gumbs and N. J. M. Horing, Plasma losses by charged particles in thin films: Effects of spatial dispersion, phonons, and magnetic field, Phys. Rev. B 43, 2119 (1991).

[32] X. Lin, I. Kaminer, X. Shi, F. Gao, Z. Yang, Z. Gao, H. Buljan, J. D. Joannopoulos, M. Soljacic, H. Chen, B. Zhang, Splashing transients of 2D plasmons launched by swift electrons, Sci. Adv. 3, e1601192 (2017).

[33] F. J. García de Abajo, Optical excitations in electron microscopy, Rev. Mod. Phys. 82, 209 (2010).

[34] F. J. García de Abajo, Multiple excitation of confined graphene plasmons by single free electrons, ACS Nano 7, 11409 (2013).

[35] X. Lin, N. Rivera, J. J. López, I. Kaminer, H. Chen, and M. Soljacic, Tailoring the energy distribution and loss of 2D plasmons, New J. Phys. 18, 105007 (2016).

[36] M. Rabaud and F. Moisy, Ship Wakes: Kelvin or Mach Angle? Phys. Rev. Lett. 110, 214503 (2013). 
[37] X. Shi, X. Lin, F. Gao, H. Xu, Z. Yang, and B. Zhang, Caustic graphene plasmons with Kelvin angle, Phys. Rev. B 92, 081404(R) (2015).

[38] F. J. García de Abajo and P. M. Echenique, Wake potential in the vicinity of a surface, Phys. Rev. B 46, 2663 (1992).

[39] R. Pethiyagoda, Scott W. McCue, and Timothy J. Moroney, What is the apparent angle of a Kelvin ship wave pattern? J. Fluids Mech. 758, 468 (2014).

[40] X. Lin, Y. Yangc, N. Rivera, J. J. López, Y. Shen, I. Kaminer, H. Chen, B. Zhange, J. D. Joannopoulosb, and M. Soljacic, All-angle negative refraction of highly squeezed plasmon and phonon polaritons in graphene-boron nitride heterostructures, Proc. Natl. Acad. Sci. USA 114, 6717 (2017).
[41] K. Akbari, Z. L. Mišković, S. Segui, J. L. Gervasoni, and N. R. Arista, Energy losses and transition radiation in multilayer graphene traversed by a fast charged particle, ACS Photon. 4, 1980 (2017).

[42] A. V. Arzhannikov and I. A. Kotelnikov, Excitation of ship waves by a submerged object: New solution to the classical problem, Phys. Rev. E 94, 023103 (2016).

[43] P. Bodenheimer, G. P. Laughlin, M. Rozyczka, T. Plewa, and H. W. Yorke, Numerical Methods in Astrophysics: An Introduction (Taylor \& Francis Group, Boca Raton, FL, 2007).

[44] P. A. D. Gonçalves and N. M. R. Peres, An Introduction to Graphene Plasmonics (World Scientific, Singapore, 2016). 